\title{
The solsticial pause on Mars: 2 Modelling and investigation of causes
}

\author{
David P. Mulholland ${ }^{\mathrm{a}, 1, *}$, Stephen R. Lewis ${ }^{\mathrm{b}}$, Peter L. Read ${ }^{\mathrm{a}}$, \\ Jean-Baptiste Madeleine ${ }^{\mathrm{c}}$ and Francois Forget ${ }^{\mathrm{c}}$ \\ ${ }^{a}$ Atmospheric, Oceanic $\&$ Planetary Physics, University of Oxford, Clarendon \\ Laboratory, Parks Road, Oxford OX1 3PU, UK \\ ${ }^{\mathrm{b}}$ Department of Physical Sciences, The Open University, Walton Hall, Milton \\ Keynes MK7 6AA, UK \\ ${ }^{\mathrm{c}}$ Laboratoire de Météorologie Dynamique (LMD), CNRS, IPSL, 4 pl. Jussieu, \\ 75252 Paris, France \\ Copyright (C) 2015 The Authors
}

Number of pages: 43

Number of tables: 1

Number of figures: 15

\footnotetext{
* Corresponding author Email address: d.p.mulholland@reading.ac.uk (David P. Mulholland).

1 Now at Department of Meteorology, University of Reading, Harry Pitt Building, Reading RG6 6AL, UK
} 


\section{Proposed Running Head:}

Modelling the martian solsticial pause

Please send Editorial Correspondence to:

David P. Mulholland

Department of Meteorology, University of Reading

Harry Pitt Building, 3 Earley Gate, Reading RG6 6AL, UK

Email: d.p.mulholland@reading.ac.uk

Telephone: (+44) 1183787762 


\section{ABSTRACT}

The martian solsticial pause, presented in a companion paper (Lewis et al., this issue), was investigated further through a series of model runs using the UK version of the LMD/UK Mars Global Climate Model. It was found that

5 the pause could not be adequately reproduced if radiatively active water ice clouds were omitted from the model. When clouds were used, along with a realistic time-dependent dust opacity distribution, a substantial minimum in near-surface transient eddy activity formed around solstice in both hemispheres. The net effect of the clouds in the model is, by altering the thermal structure of the atmosphere, to decrease the vertical shear of the westerly jet near the surface around solstice, and thus reduce baroclinic growth rates. A similar effect was seen under conditions of large dust loading, implying that northern midlatitude eddy activity will tend to become suppressed after a period of intense flushing storm formation around the northern cap edge. Suppression of baroclinic eddy generation by the barotropic component of the flow and via diabatic eddy dissipation were also investigated as possible mechanisms leading to the formation of the solsticial pause but were found not to make major contributions. Zonal variations in topography were found to be important, as their presence results in weakened transient eddies around winter solstice in both hemispheres, through modification of the near-surface flow. The zonal topographic asymmetry appears to be the primary reason for the weakness of eddy activity in the southern hemisphere relative to the northern hemisphere, and the ultimate cause of the solsticial pause in both hemispheres. The meridional topographic gradient was found to exert a much weaker influence on near-surface transient eddies.

Keywords: MARS, ATMOSPHERE; MARS, CLIMATE; ATMOSPHERES, 
DYNAMICS 


\section{Introduction}

In Lewis et al. (this issue), hereafter referred to as P1, martian transient waves were diagnosed from a reanalysis of Thermal Emission Spectrometer (TES) data. A particular feature identified in the record was the 'solsticial pause': a weakening of waves near the surface around winter solstice of each year analysed, seen in both hemispheres.

Solsticial pauses with a range of depths have been simulated by martian global climate models (MGCMs) (Hourdin et al., 1995; Basu et al., 2006; Wang et al., 2013; Kavulich Jr et al., 2013), and existing modelling literature suggests that the depth of a modelled solsticial pause is enhanced by the presence of one or both of a large solsticial dust loading (representative of a global dust storm) (Hourdin et al., 1995; Kuroda et al., 2007) and radiatively active water ice clouds (Wilson, 2011). The reduction in eddy activity under conditions of high dust loading is understood to be a result of changes to the zonal jet (Kuroda et al., 2007), and several important features of winter hemisphere eddies have been drawn from observational data (Wang, 2007), but a detailed description of the mechanisms behind the solsticial pause, which occurs during years both with and without a global dust storm, is currently lacking.

The midwinter minimum in North Pacific atmospheric storminess on Earth, though different in several ways to the martian solsticial pause (see P1), has been studied in detail in recent years, and some of the explanations put forward for the terrestrial case may be relevant to Mars. These include: an increase in barotropic damping around solstice (Deng and Mak, 2006); eddy dissipation through diabatic effects (Chang, 2001); and localised effects of interaction with 
topography (Penny et al., 2010; Park et al., 2010).

In this paper we use an MGCM to measure the net effects of several of these factors, some of which may contribute to the formation of the pause, in both hemispheres. First, in section 2, the model used is described. In section 3, the set of simulations are introduced, along with summary measures of the extent to which each is able to reproduce solsticial minima similar to those shown in P1. Section 4 analyses the contributions of various mechanisms to the formation of the solsticial pause, from seasonal variation in atmospheric baroclinicity, to other means of reducing eddy growth, and finally to the more fundamental impact of surface topography. In section 5 , the role of topography is discussed further, and our insights into the nature of the pause are used to provide explanation for its interannual variability as presented in P1. Finally, the key results of the paper are summarised in section 6 .

\section{Model description}

The model used from this study is an updated, UK version of the LMD/UK Mars Global Climate Model (UKMGCM) described in P1. This version includes a microphysical cloud scheme, which predicts ice particle sizes and growth rates taking into account temperature, humidity and local density of dust nuclei (Montmessin et al., 2004; Madeleine et al., 2012). Water vapour and ice tracers undergo parameterised turbulent diffusion, convective adjustment and gravitational sedimentation. The radiative effect of water ice clouds can be included; the radiation scheme (Madeleine et al., 2011) calculates cloud extinction depending on the ice particle size, using optical parameters from 75 Warren (1984) (see Madeleine et al. (2012) for more details). 


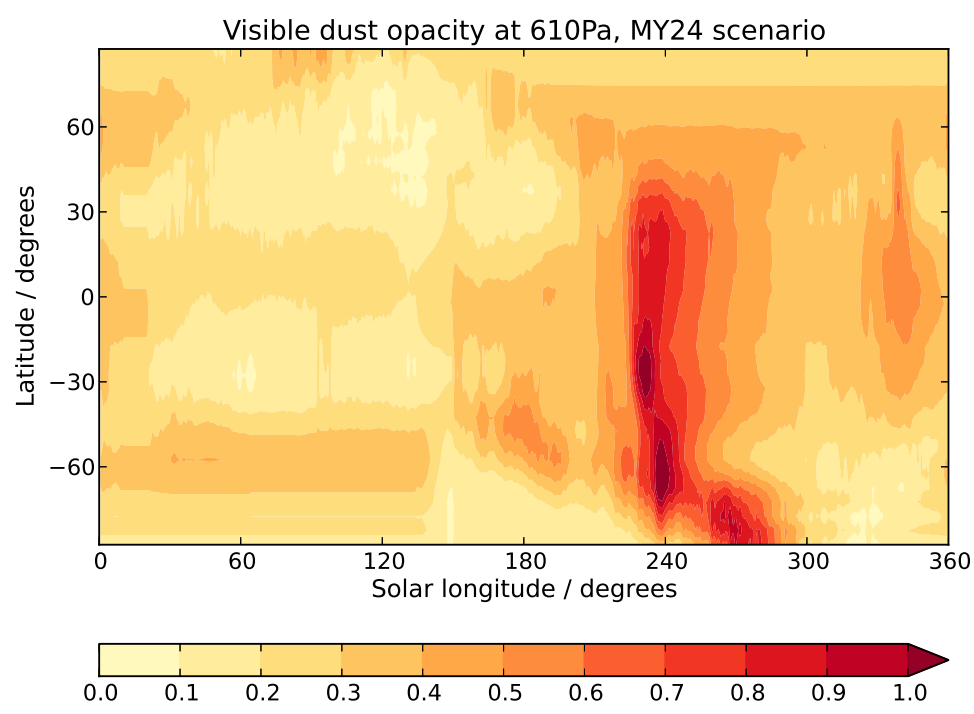

Fig. 1. The 'MY24' dust scenario, constructed from a combination of MY24 and MY25 reanalysis dust opacities, plotted as a zonal mean function of latitude and solar longitude.

Full lifting and transport simulations using radiatively active dust are possible in the UK model, but in this work, prescribed dust opacities are used in lieu of a transported dust field, to enable more direct comparisons with observations and to fix the dust opacity field within a set of controlled model experiments used to study the sensitivity of the solsticial pause to several different effects. In this mode, the dust field that the radiation scheme 'sees' is comprised of particles of radius $1.5 \mu \mathrm{m}$, with variation of mass mixing ratio with height following a Conrath profile. Mixing ratios are scaled so that the total column optical depth, calculated in two visible/near-IR bands and three thermal IR bands and using a ratio of visible to infrared opacity of 2 , fits a prescribed distribution. The visible optical depth may have a constant value in time and space, or follow a more complex spatially and/or temporally varying function (Forget et al., 1999). 
One such distribution used in this work varies with longitude, latitude and time, and replicates dust opacities from MY24 as observed by the Thermal Emission Spectrometer onboard Mars Global Surveyor (Smith et al., 2000). It was constructed using the reanalysis dataset described in P1. Since data were not available for $L_{s}=0-140^{\circ}$ in MY24, the dust opacity scenario for this year uses optical depths from MY25 of the reanalysis dataset during this period. ${ }_{95}$ The differences in dust loading between years is expected to be fairly small at this time of the martian year (northern spring/summer). The 'MY24' dust scenario is shown as a zonal mean in Fig. 1.

The key point is that model runs using the 'MY24' scenario can be compared directly to the reanalysis. Aside from possible inaccuracies in the vertical dust distribution, which will be compensated for in the reanalysis (through temperature assimilation) but not in the free-running model, any differences between model and reanalysis represent deficiencies in the model's ability to simulate correctly the atmospheric state (temperature, pressure, wind) given the correct dust opacity forcing.

Note that there is considerable uncertainty in the column dust opacity in both winter polar regions, as there were no TES opacity data available in these regions. As a result of the approach taken in the data assimilation procedure, dust opacities remain constant over a period without available observations, as can be discerned from Fig. 1. The simulations presented in the next section include the use of two alternative prescribed dust distributions, which gives information on the sensitivity to dust loading more generally, and provides some confidence that the impact of water ice clouds is not specific to the use of a particular, possibly inaccurate, polar dust distribution. 
The model runs described below were all carried out using the T31 spectral truncation, corresponding to a $3.75^{\circ} \times 3.75^{\circ}$ dynamical grid and a $5^{\circ} \times 5^{\circ}$ physics grid, the same as was used for the reanalysis of P1. The use of a freerunning MGCM complements the analysis of observational and assimilated data by allowing the isolation of the net effects of each of several physical mechanisms, through the use or neglect of each in a particular simulation. In this work, the three model forcings that were varied in this way were the dust opacity field, the radiative effects of water ice clouds and the surface topography.

\section{Model simulations}

To test whether or not the model reproduces the reanalysis as presented in $\mathrm{P} 1$, and to investigate the conditions necessary for the formation of a solsticial pause, a series of simulations were performed using, in each case, a prescribed dust opacity field. The simulations are named and summarised in Table 1. Two used the 'MY24' dust scenario described previously, one including the radiative effects of water ice clouds $\left(\tau_{M Y 24}^{*}\right)$ and the other neglecting clouds $\left(\tau_{M Y 24}\right)$. A similar pair of runs with $\left(\tau_{\text {low }}^{*}\right)$ and without $\left(\tau_{\text {low }}\right)$ clouds used instead a constant visible dust opacity of 0.2 , referenced to the $610 \mathrm{~Pa}$ pressure surface. Finally, $\tau_{\text {high }}$ simulated a large, perennial dust storm by using a constant $610 \mathrm{~Pa}$ dust opacity of 2.0 , and neglected water ice clouds.

Table 1 also includes a quantity $\frac{\left[T_{\text {max }}^{\prime}\right]_{\text {solstice }}}{\left[T_{\text {max }}^{\prime}\right]_{\text {pre } \text { post }}}$ (along with its constituent parts)

which measures, in a crude way, the extent to which a solsticial pause was produced in the northern hemisphere for a particular run. This quantity was calculated by finding the maximum value of the RMS transient temperature, 
filtered to include only signals with period between 1.5 and 30 sols, at a height of $2.5 \mathrm{~km}$ within the region $30-90^{\circ} \mathrm{N}$, at each point in time, and taking the 140 ratio of its averages over $L_{s}=240-300^{\circ}$ and over $L_{s}=\left(180-240^{\circ}, 300-360^{\circ}\right)$. Low values therefore signify a deep minimum in transient eddy activity: the reanalysis value for MY24 is 0.50 .

In summary, all runs except $\tau_{\text {low }}$ produced a discernible solsticial minimum in near-surface eddy activity. $\tau_{M Y 24}^{*}$ came closest to replicating the results of the reanalysis, though fell somewhat short. A deeper solsticial pause was produced whenever dust optical depth was increased (compare $\tau_{l o w}, \tau_{M Y 24}$ and $\tau_{\text {high }}$ ), or whenever clouds were activated. The results suggest that both the presence of water ice clouds and an increase in global dust loading close to solstice contribute to the development of a solsticial pause.

\begin{tabular}{|c|c|c|c|c|c|}
\hline Name & Dust opacity & Clouds? & {$\left[T_{\text {max }}^{\prime}\right]_{\text {solstice }}$} & {$\left[T_{\text {max }}^{\prime}\right]_{\text {pre,post }}$} & $\frac{\left[T_{\text {max }}^{\prime}\right]_{\text {solstice }}}{\left[T_{\text {max }}^{\prime}\right]_{\text {pre }, \text { post }}}$ \\
\hline$\tau_{M Y 24}$ & MY24 & No & 6.40 & 7.09 & 0.90 \\
\hline$\tau_{M Y 24}^{*}$ & MY24 & Yes & 5.55 & 7.94 & 0.70 \\
\hline$\tau_{\text {low }}$ & 0.2 & No & 7.91 & 7.42 & 1.07 \\
\hline$\tau_{l o w}^{*}$ & 0.2 & Yes & 8.39 & 9.18 & 0.91 \\
\hline$\tau_{\text {high }}$ & 2.0 & No & 5.51 & 7.27 & 0.76 \\
\hline \multicolumn{3}{|c|}{ MY24 reanalysis } & 3.35 & 6.72 & 0.50 \\
\hline
\end{tabular}

Table 1

Summary of the model simulations performed. 


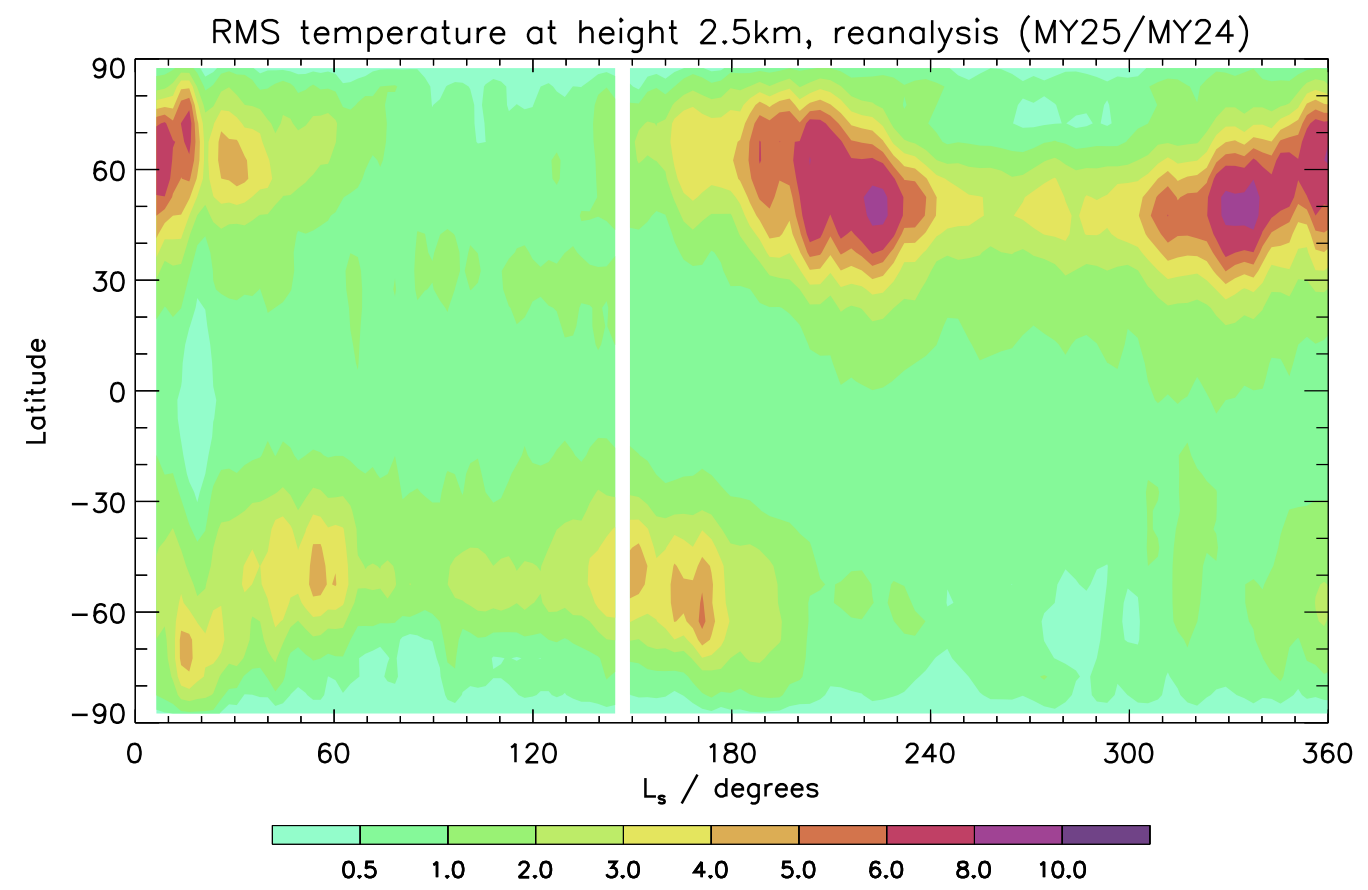

Fig. 2. RMS transient temperature $(\mathrm{K})$ at a height of $2.5 \mathrm{~km}$ above the surface, from the reanalysis. Shown is a composite of MY25 (for $L_{s}=0-140^{\circ}$ ) and MY24 $\left(L_{s}=140-360^{\circ}\right)$, which is the same split as in the 'MY24' dust scenario used below (in model simulations $\tau_{M Y 24}$ and $\tau_{M Y 24}^{*}$ ). Values are masked out for one sol around the split at $L_{s}=140^{\circ}$.

To aid comparison with model simulations, one year of zonal-mean RMS transient temperature at $2.5 \mathrm{~km}$ above the surface from the reanalysis (specifically a hybrid of MY24 and MY25, formed in the same way as the 'MY24' dust opacity scenario, described above) is displayed in Fig. 2. The same quantity for the full reanalysis record can be seen in P1, Fig. 1. In this and all subsequent plots, temporal filtering is used to retain only eddies with period between 1.5 and 30 sols, which are termed 'transient', and time series are smoothed using an averaging window of length 9 sols. The solsticial pause is clearly visible in each hemisphere, as is the stronger peak amplitude of transient eddies in the northern hemisphere than in the southern hemisphere. 
Fig. 3 (left column) shows the equivalent RMS transient temperature from model simulations $\tau_{\text {low }}$ and $\tau_{M Y 24}$, both performed without the radiative impact of water ice clouds. The constant opacity case shows no sign of a solsticial pause in the northern hemisphere, with a peak RMS of $8-9 \mathrm{~K}$ throughout the period $L_{s}=180-360^{\circ}$. With the MY24 dust scenario, a minimum in RMS develops between $L_{s}=260^{\circ}$ and $L_{s}=290^{\circ}$, but it is much smaller in amplitude and shorter in duration than that seen in the reanalysis. The overlaid meridional temperature gradients show that in both cases the peak in transient eddy activity lies around $5^{\circ}$ to the north of the latitude where the temperature gradient is strongest. Evidently the MY24 dust scenario, with elevated opacities over most of the planet from $L_{s} \sim 210^{\circ}$ onwards, goes some way towards forcing a solsticial pause, perhaps for a similar reason to that identified by Kuroda et al. (2007) for a large solsticial dust storm; however, this realistic dust loading falls well short of reproducing the results from the reanalysis for the same year. The southern hemisphere shows similar results, with fairly constant eddy activity for $\tau_{\text {low }}$ and a short (though otherwise quite realistic) minimum for $\tau_{M Y 24}$.

\subsection{With active water ice clouds $\left(\tau_{\text {low }}^{*}, \tau_{M Y 24}^{*}\right)$}

Fig. 3 (right column) shows the results of a similar pair of runs but with radiatively active clouds now included $\left(\tau_{\text {low }}^{*}\right.$ and $\left.\tau_{M Y 24}^{*}\right)$. Focusing again on the northern hemisphere, with MY24 dust opacity the solsticial pause is now wider and deeper than before, and resembles somewhat the observed pause 


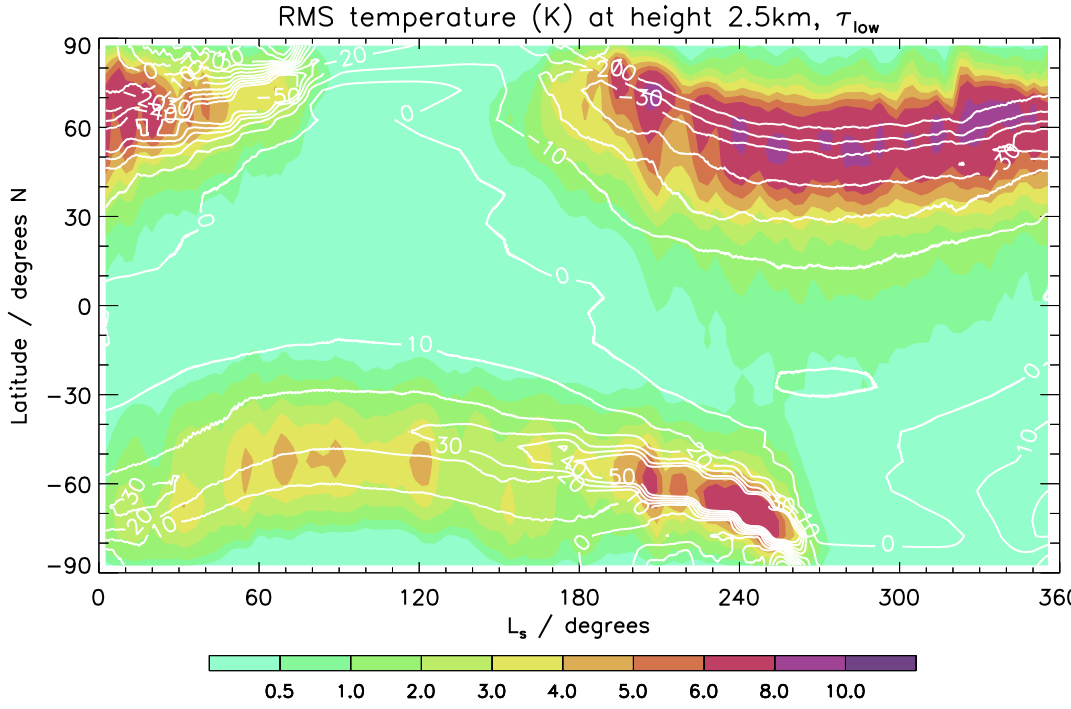

(a)

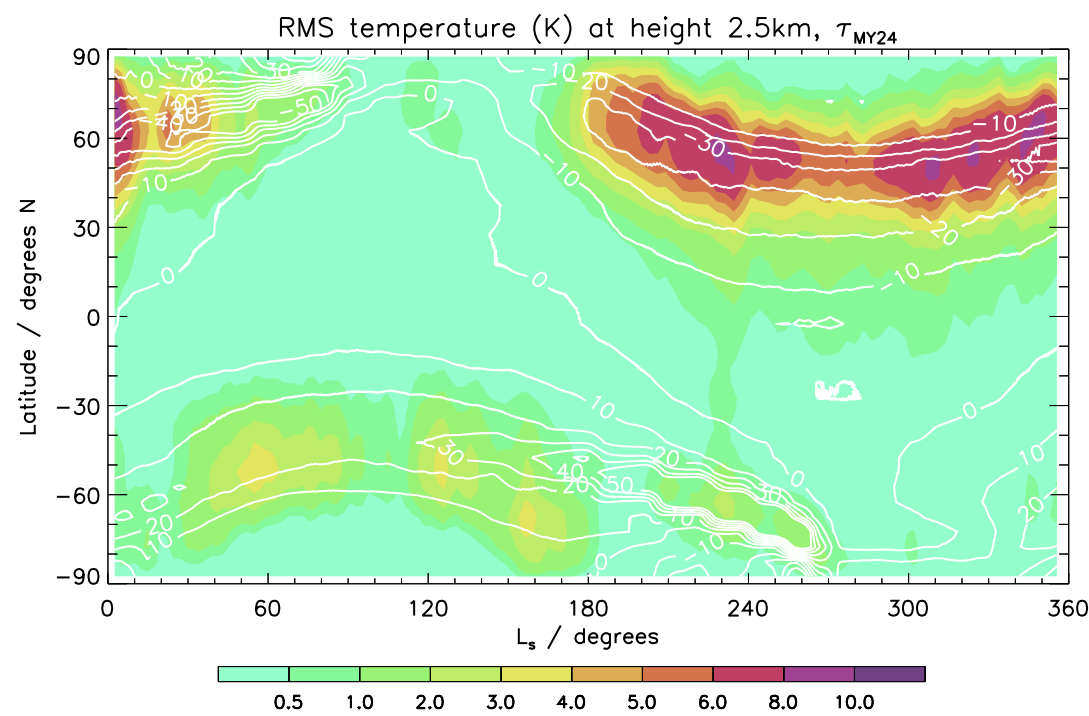

(c)

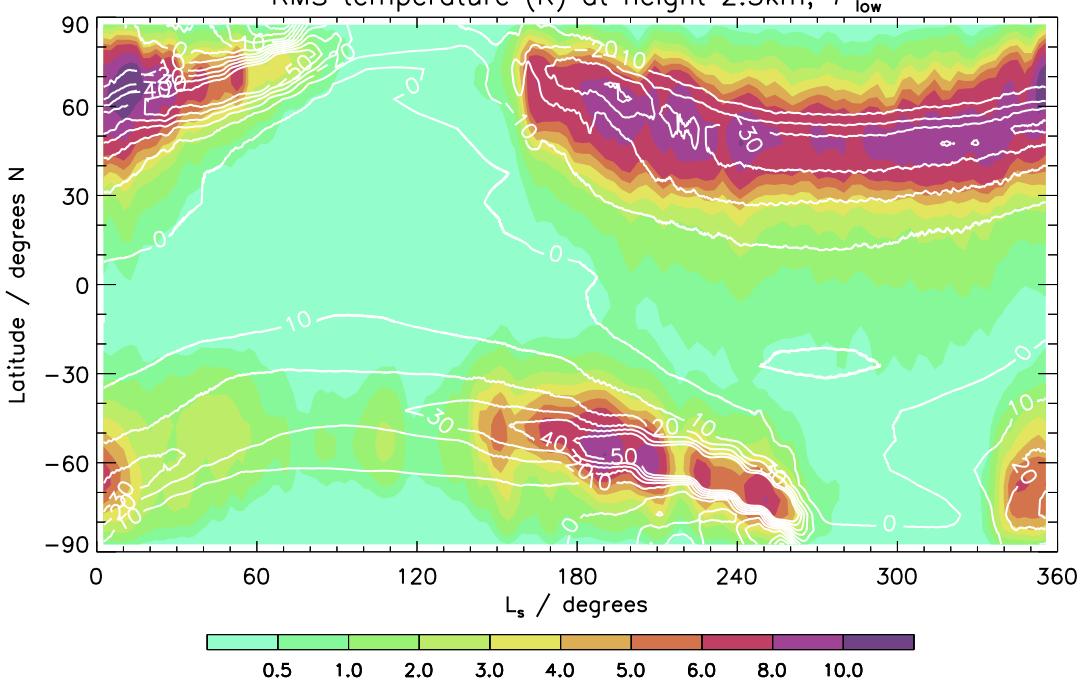

(b)

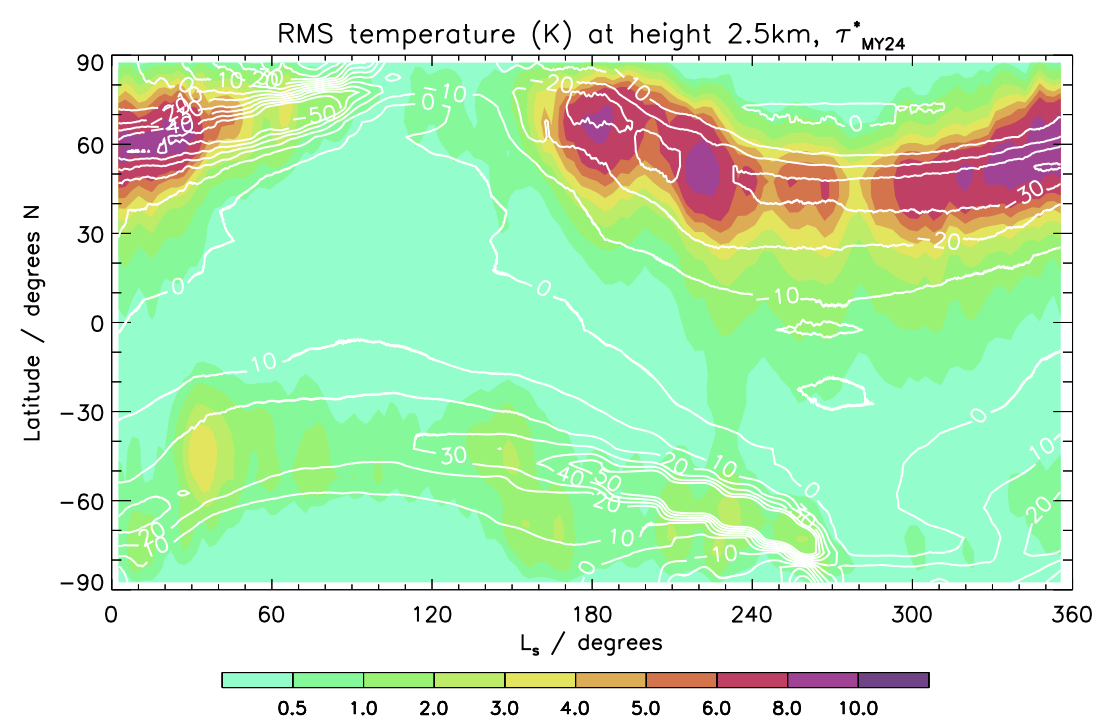

(d)

Fig. 3. RMS transient temperature (K) at a height of $2.5 \mathrm{~km}$ above the surface, as in Fig. 2, output from the MGCM from simulations (a) $\tau_{\text {low }}$, (b) $\tau_{\text {low }}^{*}$, (c) $\tau_{M Y 24}$ and (d) $\tau_{M Y 24}^{*}$ (moving from top to bottom, the more realistic dust distribution is introduced, and moving from left to right, radiatively active ice clouds are introduced). Meridional temperature gradient $\frac{d T}{d y}$ at $200 \mathrm{~m}$ above the ground $\left(10^{-6} \mathrm{Km}^{-1}\right)$ is overlaid in white contours. 
in the reanalysis, though the model pause is much less well defined (RMS temperature does not drop off sharply enough before $L_{s}=240^{\circ}$ ), and transient

eddy amplitudes are slightly larger throughout the northern autumn/winter period. Compared to $\tau_{M Y 24}$, the pause has been deepened by both increased eddy activity in early autumn $\left(L_{s}=180-220^{\circ}\right)$ and late winter $\left(320-360^{\circ}\right)$, and weakened activity around solstice. The southern hemisphere pause is also enhanced, and is now wider than that of the reanalysis. $\tau_{\text {low }}^{*}$ also simulates a solsticial pause in the southern hemisphere, and a weak pause in the northern hemisphere. Particularly in the pre-solstice period in $\tau_{\text {low }}^{*}$, and throughout $L_{s} \approx 180-300^{\circ}$ in $\tau_{M Y 24}^{*}$, the peak in eddy activity is now located at or to the south of the latitude of strongest temperature gradient. In $\tau_{M Y 24}^{*}$, around solstice, the location of the peak temperature gradient is roughly unchanged from $\tau_{M Y 24}$, meaning that the peak in eddy activity is shifted southward by $\sim 5^{\circ}$, compared to $\tau_{M Y 24}$.

For $\tau_{M Y 24}^{*}$, zonal wavenumbers 1-3 are all reduced to similar levels around solstice. Transitions between the various modes occur during the autumn/winter season. $s=1$ perturbations tend to be confined north of $45^{\circ} \mathrm{N}$, while $s=3$ are located south of $60^{\circ} \mathrm{N}$, similar to what was seen in MY26 by Wang (2007).

The cloud ice mixing ratios produced in simulation $\tau_{M Y 24}^{*}$ are shown in Fig. 4. Polar hood clouds are confined below $100 \mathrm{~Pa}$, and are generally thickest near the seasonal cap edge (which, for reference, is located at approximately $\left(60^{\circ}\right.$, $\left.50^{\circ}, 50^{\circ}\right) \mathrm{N}$ at $\left.L_{s}=\left(220^{\circ}, 270^{\circ}, 320^{\circ}\right)\right)$. Cloud optical depth in this model run reaches a maximum around the time of a regional dust storm at $L_{s}=240^{\circ}$, and declines thereafter. It is also notable that cloud optical depth briefly drops to a minimum at $L_{s} \approx 200^{\circ}$ (not shown), coincident with the gap between the two pre-solstice peaks seen in Fig. 3(d). 

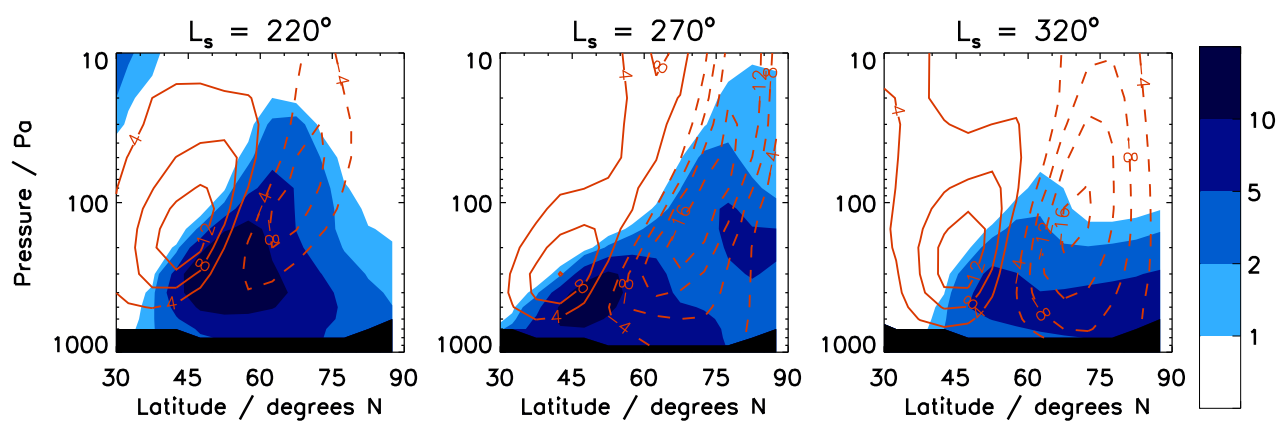

Fig. 4. Change in $\bar{u},\left(\tau_{M Y 24}^{*}-\tau_{M Y 24}\right)\left(\mathrm{m} \mathrm{s}^{-1}\right.$, contours, positive values solid, negative values dashed), and water ice cloud mixing ratio for $\tau_{M Y 24}^{*}\left(10^{-6} \mathrm{~kg} / \mathrm{kg}\right.$, shading). The surface is coloured in black.

Compared to observations, column ice opacities in northern winter mid- and

high latitudes are somewhat overestimated by the model: visible ice opacities in the model north polar hood are typically $0.2-0.5$, and reach values of around 1 at their peak at $L_{s} \approx 240^{\circ}$, whereas observations from Mars Climate Sounder (McCleese et al., 2007; Benson et al., 2011) found that typical north polar hood visible opacities are around 0.1-0.3. Therefore, the thermodynamical impact of the clouds (seen in the change to the zonal wind, drawn in Fig. 4, and discussed further in the next section) may be greater in the UKMGCM than it is in reality, and so quantitative aspects of some of the results shown in the next section should be considered as specific to the UKMGCM. The qualitative robustness of the impact of water ice clouds on the solsticial pause is discussed in section 5 . 


\section{Mechanisms behind the pause}

\subsection{Changes in atmospheric baroclinicity}

Having found that the inclusion or removal of water ice clouds strongly affects the ability of the MGCM to simulate the solsticial pause, the model runs were analysed more closely to determine the manner in which the clouds exert their influence. The most obvious mechanism for this is that the clouds alter the structure of the atmosphere so as to reduce baroclinicity at low levels around solstice.

\subsubsection{Basic atmospheric state}
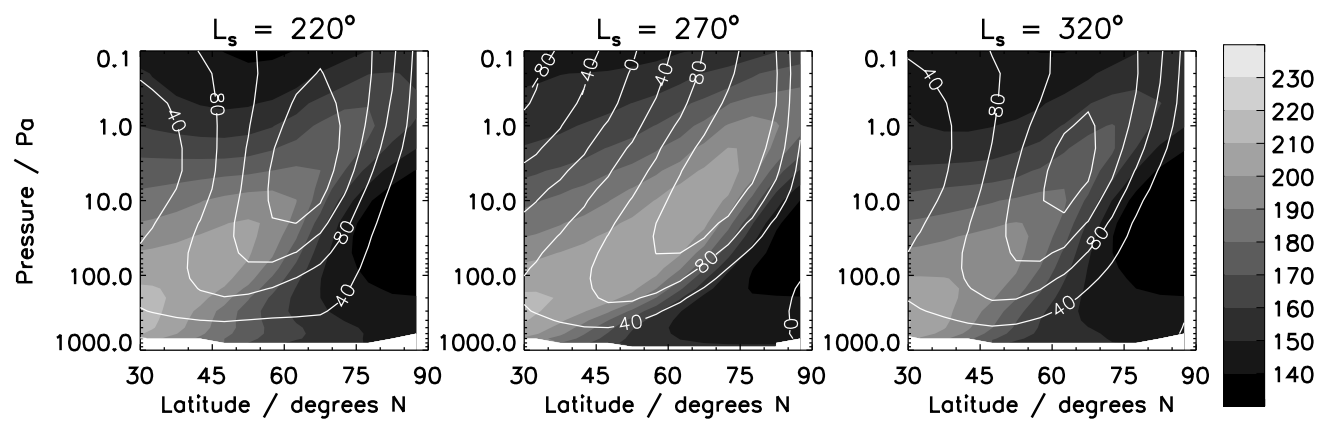

Fig. 5. Zonal-mean temperature $\bar{T}$ (K, shading) and zonal wind $\bar{u}\left(\mathrm{~m} \mathrm{~s}^{-1}\right.$, contours) for $\tau_{M Y 24}^{*}$

Fig. 5 shows the zonal-mean state of $\tau_{M Y 24}^{*}$, the most realistic of the model runs. The change to the zonal-mean zonal wind below $10 \mathrm{~Pa}$ caused by the addition of water ice clouds can be seen in Fig. 4. The addition of clouds slows the zonal wind by $5-10 \mathrm{~m} \mathrm{~s}^{-1}$ near the ground at $60-80^{\circ} \mathrm{N}$ around solstice, and below $100 \mathrm{~Pa}$ the jet is shifted equatorward in such a way as to enhance the poleward tilt of this lower portion. The change to the thermal structure 
near the ground around solstice encourages this, as temperature contours at $40-70^{\circ} \mathrm{N}$ (Fig. 5) are roughly aligned with the vertical before and after solstice but slope strongly around solstice as the surface at $40-70^{\circ} \mathrm{N}$ cools. Since cloud formation is determined primarily by atmospheric temperature (Benson et al., 2011), the clouds adopt approximately the same sloped structure around solstice (Fig. 4). A cooling of up to $5-10 \mathrm{~K}$ occurs in the vicinity of and below the clouds, the effect of which is to reduce the meridional temperature gradient on the clouds' poleward side, and strengthen it on the equatorward side. The clouds do, therefore, alter the mean state of the atmosphere, and so have the potential to affect baroclinic instability.

In the MY24 reanalysis, the reduction in zonal wind near the surface at 60$80^{\circ} \mathrm{N}$ begins earlier in the year than it does in $\tau_{M Y 24}^{*}$, along with the more rapid reduction in eddy activity seen previously (compare Figs. 2 and 3(d) at $L_{s} \approx 230^{\circ}$.).

\subsubsection{Near-surface temperature gradients}

A comparison of the left and right columns of Fig. 3 shows that the maximum (most negative) meridional temperature gradient very close to the ground in the northern hemisphere is weakened by the addition of clouds (seen particularly during the pre-solstice period), since the peak in cloud opacity tends to lie to the south of the peak temperature gradient, implying a reduction to the peak temperature gradient (on the clouds' poleward side) as explained above. The latitude of the peak gradient is also shifted $\sim 5^{\circ}$ to the north before solstice in $\tau_{M Y 24}^{*}$ relative to $\tau_{M Y 24}$, but reaches a similar latitude of $\sim 45-50^{\circ} \mathrm{N}$ at solstice in both $\tau_{M Y 24}$ and $\tau_{M Y 24}^{*}$, in good agreement with the reanalysis. 
Transient eddy activity does not closely follow the strength of the temperature gradient; for example, compare the very similar gradients seen in southern midlatitudes at $L_{s}=120-210^{\circ}$ in all four simulations shown in Fig. 3, with the different temperature variances that result. The reanalysis itself (not shown) features a northern midlatitudes temperature gradient at height $200 \mathrm{~m}$ that is fairly constant over $L_{s}=220-310^{\circ}$, and is stronger at solstice than at the time of the pre-solstice maximum $\left(L_{s}=200-220^{\circ}\right)$, though it does increase around the time of the post-solstice maximum. Therefore, near-surface baroclinic eddy activity cannot simply be explained in terms of the varying magnitude of the near-surface temperature gradients.

\subsubsection{Linear growth rates}

A better measure of the potential for baroclinic instability of the lower atmosphere is provided by the Eady growth rate, $\sigma$ (e.g. James and Gray (1986)):

$$
\sigma=0.31 \frac{f}{N} \frac{\partial \bar{u}}{\partial z}
$$

where $f$ is the Coriolis parameter, $N$ is the atmospheric static stability and $\bar{u}$ is the zonal-mean zonal wind velocity. Since the baroclinic zone moves equatorward from autumn to winter with the growth of the seasonal $\mathrm{CO}_{2}$ cap, the change in $f(\propto \sin$ (latitude) $)$ will cause $\sigma$ to decrease by a factor of $\sim 1.3$ from $70^{\circ}$ to $45^{\circ}$ latitude. In addition, Wang et al. (2005) found that in the TES data, $N^{2}$, at a height of $2.5 \mathrm{~km}$ above the north polar cap edge, peaked at $L_{s}=260^{\circ}$, at around twice its value at $L_{s}=200^{\circ}$, implying an increase in $N$ by a factor of $\sim 1.4$ from autumn to winter (and these results were fairly well reproduced by the model, both with and without ice clouds). Therefore, simply following the edge of the seasonal polar cap, basic state conditions 

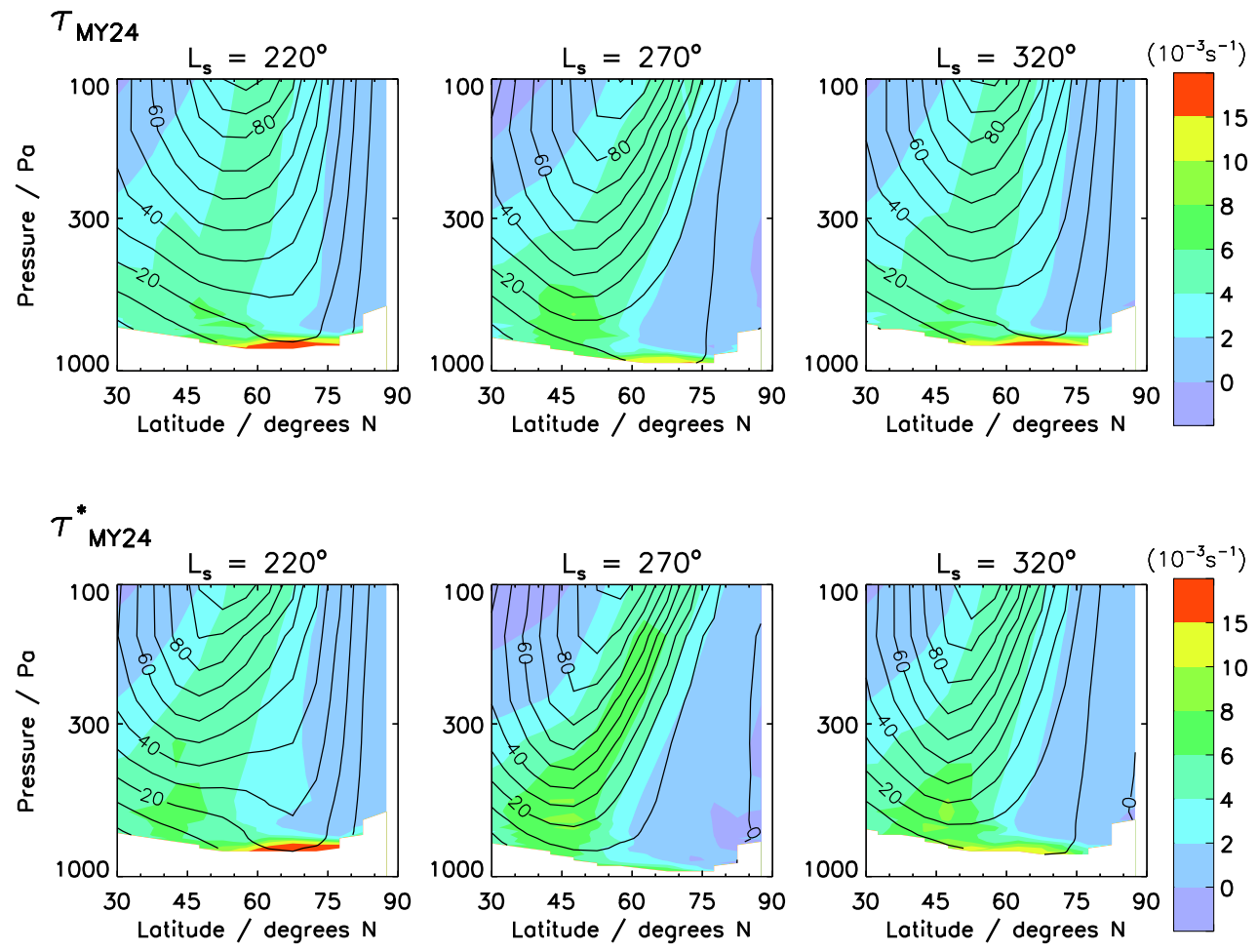

Fig. 6. Zonal mean vertical wind shear $\frac{\partial \bar{u}}{\partial z}$ (shading, $\mathrm{s}^{-1}$ ) and zonal wind $\bar{u}$, (contours, $\mathrm{m} \mathrm{s}^{-1}$ ) near the surface, for runs $\tau_{M Y 24}$ (top) and $\tau_{M Y 24}^{*}$ (bottom).

suggest a reduction in baroclinic growth rate of around $40 \%$ from autumn to winter solstice. However, the vertical wind shear, $\frac{\partial \bar{u}}{\partial z}$, has a crucial role in determining the growth rates that ultimately occur before and at solstice.

The growth rate in $\tau_{M Y 24}^{*}$ exhibits a maximum around solstice at $20 \mathrm{~Pa}$, $\sim 70^{\circ} \mathrm{N}$, in agreement with the simulated middle atmosphere RMS temperature peak (not shown). The addition of clouds, most importantly at high altitudes in the tropics, increases this upper maximum in comparison with $\tau_{M Y 24}$, by strengthening the principal meridional overturning cell (PMOC), and increasing the polar warming and the associated vertical wind shear. Growth rate $\sigma$ also maximises at solstice at $\sim 50^{\circ} \mathrm{N}$ at altitudes as low as $400 \mathrm{~Pa}$. 
However, the growth rates that are important with respect to the solsticial pause are situated very close to the ground, within the lowest few hundred metres, and vary partly due to changes in vertical wind shear. Without clouds $\left(\tau_{M Y 24}\right)$, a relatively large surface wind shear of $\geq 0.01 \mathrm{~s}^{-1}$ exists at $60-80^{\circ} \mathrm{N}$ throughout the period shown (Fig. 6 (top)). It is slightly reduced around solstice, compared to its values in late autumn and early spring. When clouds are used, this reduction in shear around solstice is more substantial, to $\approx$ $0.006 \mathrm{~s}^{-1}$ (Fig. 6 (bottom)). The jet also appears sharper and tilted further from the vertical around $100 \mathrm{~Pa}$, with an equatorward shift closer to the ground (note in particular the shift in the $10 \mathrm{~m} \mathrm{~s}^{-1}$ contour), at $L_{s}=270^{\circ}$.

This correlation between Eady growth rate and RMS transient eddy temperature (which was also noted by Wang et al. (2013), using a slightly different baroclinic parameter) is clearly displayed in Fig. 7, which shows the maximum values of the two quantities (growth rate and RMS temperature at $200 \mathrm{~m}$ and $2.5 \mathrm{~km}$ above the surface, respectively) within $30-90^{\circ} \mathrm{N}$, along with the growth rate (at $200 \mathrm{~m}$ height) at the location of the eddy temperature maximum. For $\tau_{M Y 24}^{*}$ (middle panel), the peak RMS temperature (solid line) in the northern domain shows a correlation with the maximum Eady growth rate (dashed line), and both fall to half their $L_{s}=210^{\circ}$ values by $L_{s}=280^{\circ}$. However, the locations of the two maxima differ somewhat after $L_{s} \approx 210^{\circ}$. As can be inferred from Fig. 6 (bottom panel), the peak Eady growth rate is found at $60-80^{\circ} \mathrm{N}$ throughout the season, whereas the maximum in RMS temperature is centred south of $60^{\circ} \mathrm{N}$ after about $L_{s}=210^{\circ}$. The post-solstice increase in eddy activity $\left(L_{s}=290-350^{\circ}\right)$ is not matched by a significant local rise in growth rate (dotted line), but rather by an increase in the growth rate to the north of this location, at $\sim 70^{\circ} \mathrm{N}$. 

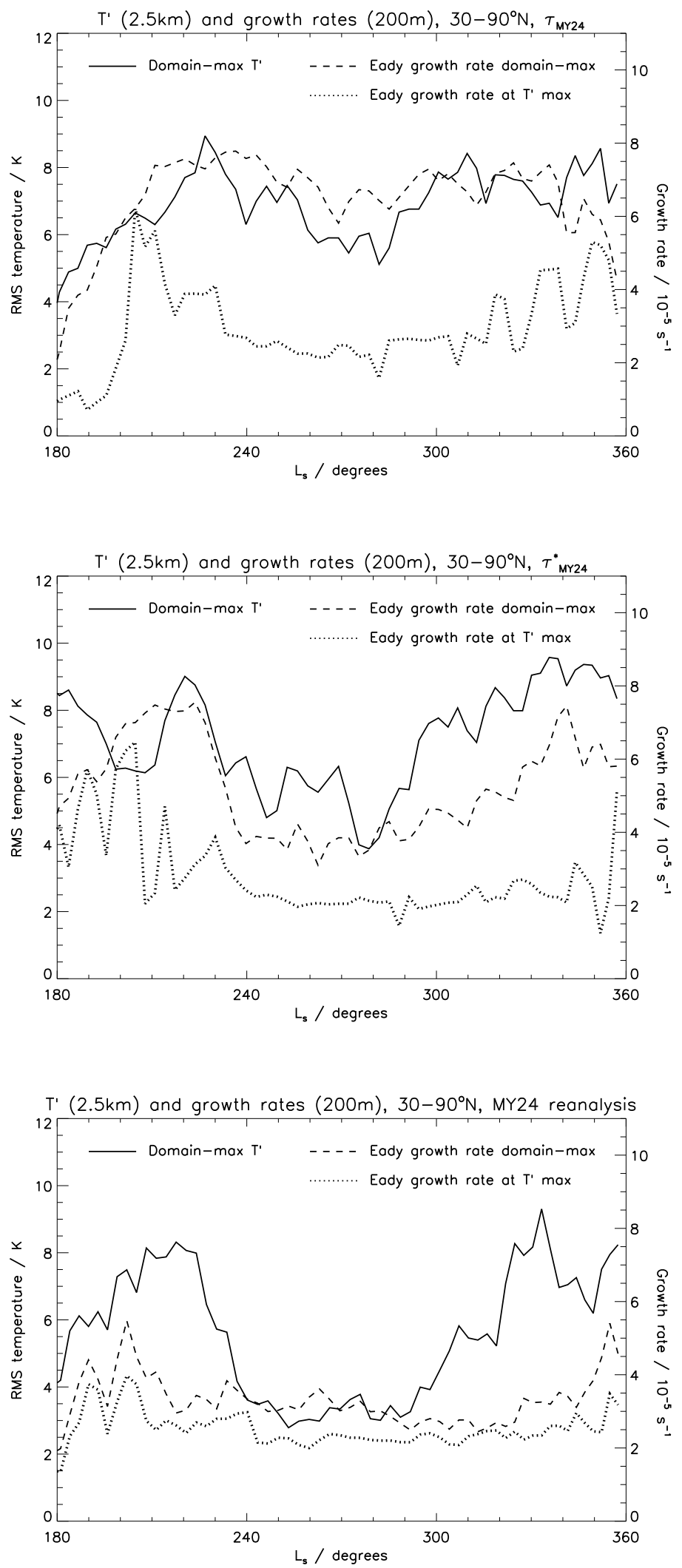

Fig. 7. Peak values of the zonal-mean RMS transient eddy temperature at $2.5 \mathrm{~km}$ height (solid, left axis) and Eady growth rate at $200 \mathrm{~m}$ height (dashed, right axis) within $30-90^{\circ} \mathrm{N}$, and zonal-mean $200 \mathrm{~m}$ Eady growth rate at the latitude of the peak in RMS temperature (dotted, right axis), during northern autumn and winter, for $\tau_{M Y 24}$ (top), $\tau_{M Y 24}^{*}$ (middle) and the reanalysis of MY24 (bottom). 
The same plot for $\tau_{M Y 24}$ (top panel) shows that its more moderate solsticial RMS temperature reduction is accompanied by reductions in both local and domain-maximum Eady growth rates. The local growth rate at solstice again falls to less than half of its pre-solstice value, suggesting that the maximum RMS temperature, which only decreases to around two thirds of its pre-solstice value, is to some extent being maintained by the presence of a larger growth rate, situated to the north, which displays only a slight solsticial minimum. This could be possible through equatorward propagation of disturbances from $60-70^{\circ} \mathrm{N}$, since the meridional flow near the surface is relatively strong (along the topographic channels) in the autumn period, as evidenced by the propagation of flushing dust storms in the same direction.

In the reanalysis (bottom panel), the situation appears more complicated, and RMS temperature peaks are quantitatively less well predicted by the Eady growth rate. While the maximum RMS temperature falls to less than half of its peak value at solstice, the local growth rate decreases by a smaller fraction. The latitude of peak growth rate coincides more closely with peak eddy activity than it does in the model (seen in the similarity of the dashed and dotted lines), as peak growth rates move southward to $\sim 60^{\circ} \mathrm{N}$ over $L_{s}=220$ $335330^{\circ}$. The post-solstice rise in RMS temperature after $L_{s}=320^{\circ}$ is matched by a modest rise in growth rate, as the $\mathrm{CO}_{2}$ cap begins to recede and the latitude of peak eddy activity moves slightly northward. It should be noted that the quasi-geostrophic assumption behind the Eady growth rate is likely to be tested at such low altitudes, within the turbulent planetary boundary layer. Nevertheless, these results may suggest that the real situation is more complex than the dynamics being simulated by the MGCM, even when ice clouds are included. 

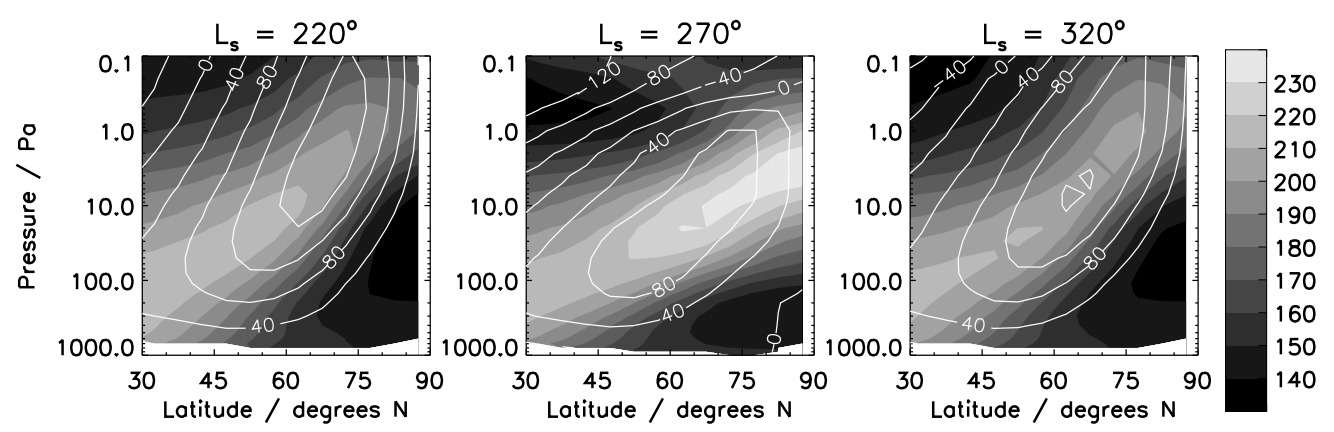

Fig. 8. As the top panel of Fig. 5, but for $\tau_{\text {high }}$.

\subsubsection{Similarities with 'dust storm' simulation ( $\tau_{\text {high }}$ )}

Similar results were observed in the dust storm simulation $\tau_{\text {high }}$, which pro-

duced a significant northern hemisphere pause (see Table 1) despite neglecting water ice clouds. As shown in other studies, an increased dust loading enhances the mean meridional circulation and strongly increases the polar warming over northern high latitudes (as much as $60 \mathrm{~K}$ warming at the $10 \mathrm{~Pa}$ level, in comparison to $\tau_{\text {low }}$ ) (Fig. 8). This results in a stronger tilt of the entire jet, which leads to a reduction in vertical shear near the surface at $60^{\circ} \mathrm{N}$, similar to that in $\tau_{M Y 24}^{*}$. It is at winter solstice that the PMOC is strongest and the tilt of the jet most pronounced, and thus the instability at the ground is strongly reduced. $s=1$ waves are most strongly affected, and $s \geq 3$ less so, giving a decrease in the dominant wavelength, in agreement with Barnes et al. (1993) and Kuroda et al. (2007).

In this simulation the variation in peak RMS temperature is well matched by the local Eady growth rate, as both drop to around one third of their pre-solstice values at solstice (Fig. 9). The maximum growth rate within the domain also reaches a minimum, at $L_{s}=280^{\circ}$, of around one third of its pre- 

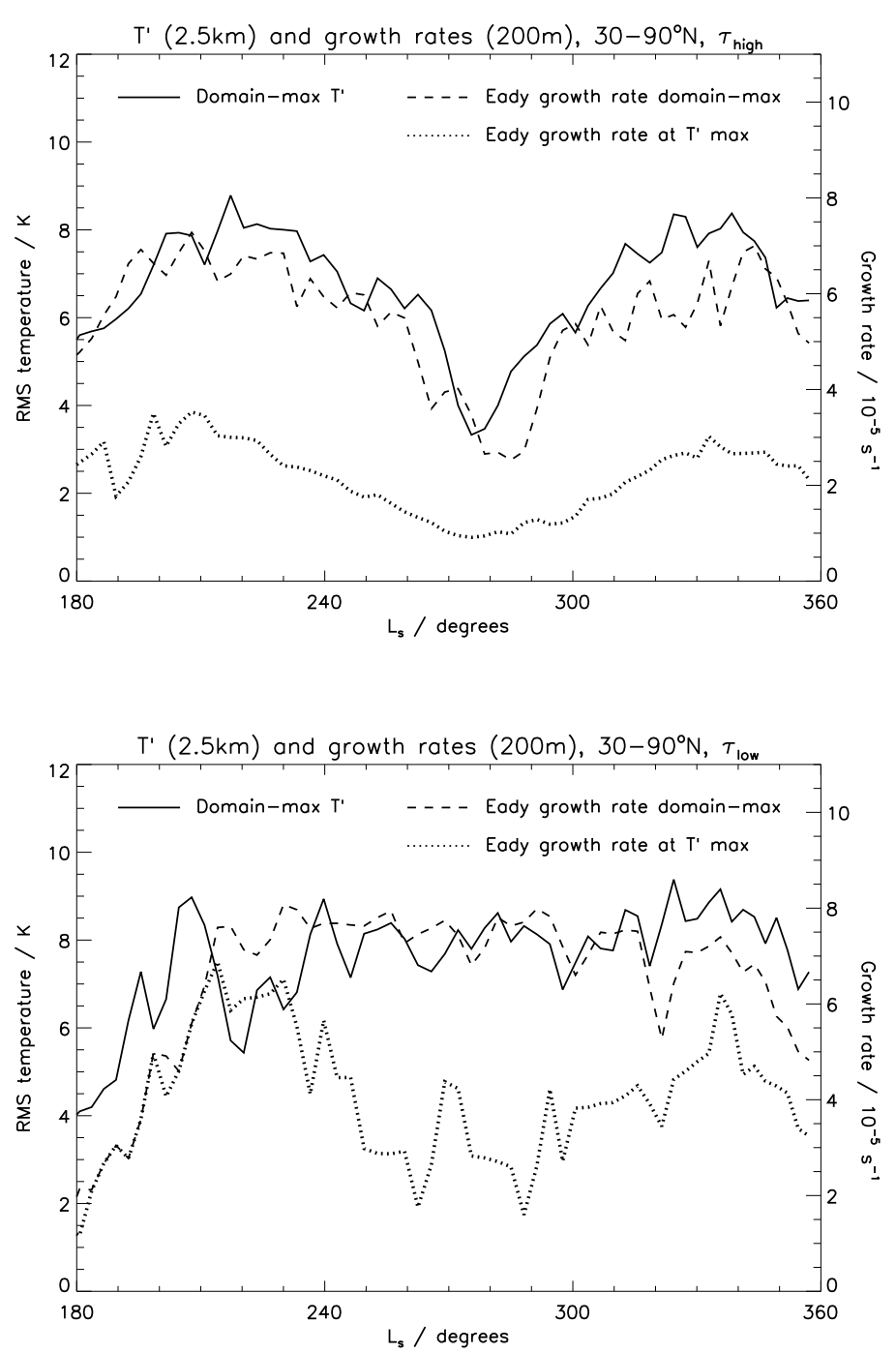

Fig. 9. As in Fig. 7, but for $\tau_{\text {high }}$ (top) and $\tau_{\text {low }}$ (bottom).

360 growth rate does not lead to any reduction in peak eddy temperature. Meanwhile, the maximum growth rate in the domain reaches a seasonal maximum around solstice. The differences between $\tau_{\text {low }}$ and $\tau_{\text {high }}$ here support the idea that consistently high growth rates at higher latitudes can contribute to the maintenance of eddy activity in the midlatitudes.

To summarise the findings of the last two subsections, then, in each of $\tau_{M Y 24}^{*}$, 


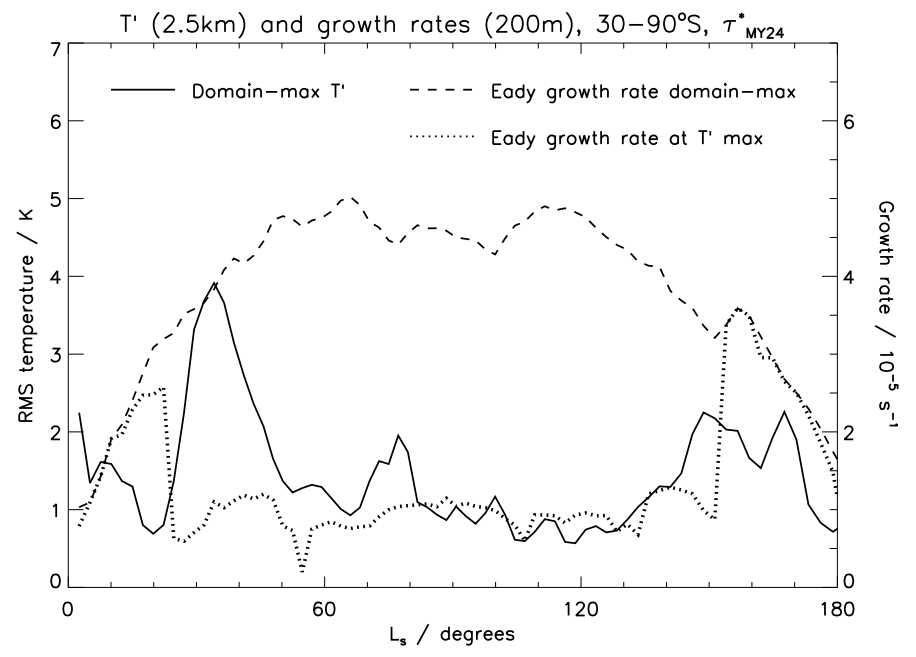

Fig. 10. As Fig. 7, but for $30-90^{\circ} \mathrm{S}$ during southern autumn and winter, in simulation $\tau_{M Y 24}^{*}$

$\tau_{M Y 24}$ and $\tau_{\text {high }}$ (as well as $\tau_{\text {low }}^{*}$, not shown), the simulated solsticial pause forms due to a meridional tilting of the westerly jet: for the dust storm case this affects the whole jet and is due to the enhanced seasonal expansion of the PMOC, whereas for the more typical non-storm case, the equatorward shearing occurs primarily near the ground, and is greatly enhanced (in the model) by the presence of the polar hood clouds. In both cases, vertical wind shear and eddy growth rates at $60-80^{\circ} \mathrm{N}$ are affected, which leads to changes in eddy activity to the south of $60^{\circ} \mathrm{N}$.

\subsubsection{Southern hemisphere}

The southern hemisphere winter jet, though weaker than in the north, also exhibits a poleward tilt over its full height, which is significant over $L_{s} \approx 30$ $150^{\circ}$. A strong reduction in windspeed poleward of $60^{\circ} \mathrm{S}$ near the ground is seen in the model at this time, and it is slightly enhanced when clouds are ${ }_{380}$ included. Clouds also reduce $\bar{u}$ and $\frac{\partial \bar{u}}{\partial z}$ at the ground at $40-50^{\circ} \mathrm{S}$, giving a 
deeper solsticial pause in $\tau_{M Y 24}^{*}$ than in $\tau_{M Y 24}$ (Fig. 3). By contrast, the wind shear does not weaken around solstice in $\tau_{l o w}$, and no pause is produced.

The southern hemisphere minimum in $\tau_{M Y 24}^{*}$ is seen despite consistently large Eady growth rates at $\sim 70^{\circ} \mathrm{S}$ (Fig. 10, dashed line), in contrast to what was 385 seen in the northern hemisphere of $\tau_{\text {low }}$ (Fig. 9). Variation in RMS temperature is in rough agreement with the local growth rate (Fig. 10, dotted line), as at solstice both fall to around one third of their pre-solstice values, and both show post-solstice increases. The fact that, unlike in the northern hemisphere, the presence of larger Eady growth rates at higher latitudes does not sustain eddy activity around solstice could perhaps be explained by the greater separation in latitude between the south polar cap edge and the peak growth rate region that exists around solstice (during $L_{s}=60-140^{\circ}$, the peak temperature variance is located at $30-40^{\circ} \mathrm{S}\left(\right.$ c.f. $\left.40-50^{\circ} \mathrm{N}\right)$, while the peak in growth rate remains at $60-70^{\circ} \mathrm{S}$ ) or by the existence of less favourable conditions for the equatorward propagation of disturbances. The baroclinic zone is much closer to the strong $60-70^{\circ} \mathrm{S}$ growth rates during the pre- and post-solstice RMS temperature maxima, due to the smaller polar cap that exists away from solstice.

In the reanalysis of southern autumn and winter of MY25 (not shown), there is a more straightforward link between growth rate and eddy activity, as the largest Eady growth rate remains close to the location of strongest eddy activity, and this growth rate agrees well with the resulting RMS temperature. The large solsticial values seen in Fig. 10 are not found in the reanalysis, perhaps because the peak moves equatorward rather than remaining at $60-70^{\circ} \mathrm{S}$ (see subsection 4.3$)$. 


\subsection{Other eddy energy sources/sinks}

In addition to the change to the basic state baroclinicity caused by the presence of water ice clouds, two other mechanisms were investigated, each of which could potentially affect the availability of transient eddy energy in the autumn and winter seasons, by introducing additional source or sink terms.

\subsubsection{Barotropic shear}

On Earth, Deng and Mak (2006) suggested that the Pacific midwinter suppression can be partly explained by barotropic eddy dissipation. On Mars, transient eddy energy is generated barotropically, and especially so around 415 solstice (see P1, Fig. 6), which would appear to rule this out as a factor in the solsticial pause. However, there is a possible indirect route for barotropic shear to contribute to the suppression of near-surface eddies.

James and Gray (1986) noted that flow with a substantial barotropic component can become significantly stabilised with respect to baroclinic instability, due to deformation of any disturbances which should occur at a rate proportional to the horizontal wind shear, $\bar{u}_{y}$. By comparing this rate to the expected linear (Eady) growth rate, they suggested that instability is significantly reduced when

$$
\frac{N \bar{u}_{y}}{f \bar{u}_{z}} \gtrsim 0.31
$$

James and Gray envisaged the barotropic governor as converting eddy kinetic energy (EKE) into zonal mean kinetic energy, and additionally reducing the

rate of baroclinic conversion from eddy available potential energy (APE) to EKE. Model EKE generation rates for $\tau_{M Y 24}$ and $\tau_{M Y 24}^{*}$ (not shown) reveal 

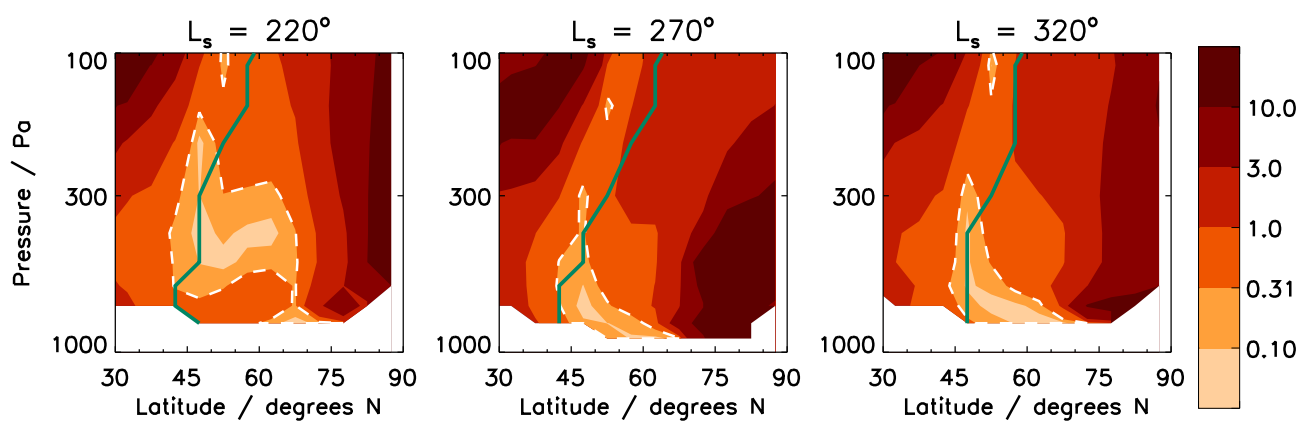

Fig. 11. The dimensionless quantity $\frac{N \bar{u}_{y}}{f \bar{u}_{z}}$, calculated for simulation $\tau_{M Y 24}^{*}$, during autumn/winter in the northern hemisphere. The dashed white contour shows where this quantity is equal to 0.31 , and the solid line marks the latitude of the strongest meridional temperature gradient at each model height level. The volume within the 0.31 contour should be 'protected' from the barotropic governor's effect.

that barotropic conversion peaks around winter solstice in both cases, in the middle atmosphere and to a lesser degree close to the surface. Both the solsticial peak in barotropic generation and the minimum in baroclinic generation are amplified by the addition of clouds, suggesting a possible causal link between the two via the barotropic governor effect.

Fig. 11 shows that large portions of the lower atmosphere (those outside the dashed region) do satisfy condition (1), but at the latitude of strongest temperature gradient it is generally not satisfied near the surface, implying that the most unstable latitudes remain, to some extent, protected from any suppressive influence of the barotropic wind shear. Although the volume of the atmosphere in which the condition is not satisfied decreases around solstice, it remains largely intact at the surface. The seasonal variation of this volume, and of its position with respect to the peak temperature gradient, is very similar in the cloud and no-cloud (not shown) cases, which display rather different 
solsticial pauses. Therefore, it would seem that the barotropic governor is not primarily responsible for the suppression near the surface during winter. An increase in the barotropicity of the atmosphere around solstice does seem to be important — it is seen consistently in each simulation that produced a solsticial pause, caused either by the presence of clouds or by a dust-enhanced circulation around solstice - but the transition from baroclinic to barotropic conversion in the lower atmosphere can be seen in the reduction of vertical wind shear as presented in the previous subsection, and it does not seem necessary to invoke any additional suppression to explain the observed reduction in eddy activity.

\subsubsection{Diabatic dissipation}

Chang (2001) found from a modelling study that a contribution to the midwinter suppression on Earth could be provided by a diabatic dissipation of eddy APE, caused by surface sensible heat flux. Away from winter solstice, he found that stronger generation of eddy APE from condensational heating was enough to overwhelm the dissipation by the surface heat flux, thereby increasing storminess.

Fig. 12 shows the rate of generation of eddy APE in the northern hemisphere of the two 'MY24' simulations, due to diabatic heating and through baroclinic conversion from zonal-mean APE. The diabatic generation rate is (following Peixoto and Oort (1992)) $\int \Gamma \overline{Q^{\prime} T^{\prime}} d m$, where $Q^{\prime}$ is the eddy diabatic heating rate and $\Gamma$ is an inverse static stability parameter defined as $\Gamma=-\left(\frac{\kappa \theta}{p T}\right)\left(\frac{\partial \tilde{\theta}}{\partial p}\right)^{-1}$, where $\tilde{\theta}$ is the domain-mean potential temperature on a particular pressure surface. The rate includes contributions from direct heat- 


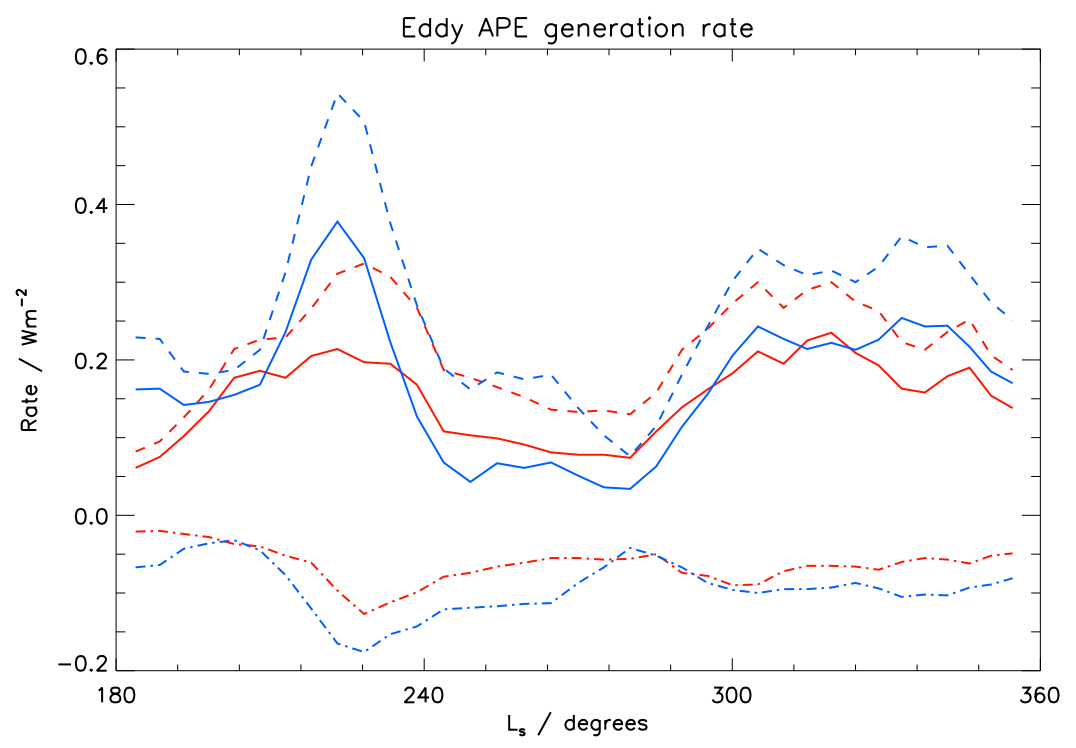

Fig. 12. Eddy available potential energy generation rates (in $\mathrm{W} \mathrm{m}^{-2}$ ), vertically integrated and averaged over $30-90^{\circ} \mathrm{N}$, for $\tau_{M Y 24}$ (red) and $\tau_{M Y 24}^{*}$ (blue). Lines show contributions from baroclinic conversion (dashed) and diabatic heating (dash-dotted), and their sum (solid).

ing by dust, $\mathrm{CO}_{2}$ and water ice clouds, and the surface sensible heat flux. The contribution from latent heat of condensation is several orders of magnitudes smaller, so is neglected. In both cases, diabatic effects (dash-dotted lines) dissipate eddy APE throughout the autumn/winter period, meaning that perturbations in radiative cooling are positively correlated with positive temperature perturbations, and thus act to damp baroclinic waves. Since most of the winter polar atmosphere receives little or no solar flux, this reflects the fact that warm regions tend to cool faster than colder regions.

Diabatic dissipation of eddy APE acts in opposition to, and primarily varies in response to, its generation baroclinically from zonal-mean APE (dashed lines), which peaks before and after solstice in both cases. The net result (generation - dissipation, solid lines) shows seasonal progressions that agree 
with the respective near-surface temperature variances, with a lower net APE generation rate over $L_{s}=240-300^{\circ}$ for $\tau_{M Y 24}^{*}$. Over $\sim 240-270^{\circ}$, baroclinic conversion is at least as rapid for $\tau_{M Y 24}^{*}$ as for $\tau_{M Y 24}$, and the lower net rate 475 is due to greater diabatic dissipation. However, for $\sim 270-300^{\circ}$, the lower net rate for $\tau_{M Y 24}^{*}$ is the result of reduced baroclinic conversion. From these domain-average figures, it appears that thermal damping caused by water ice clouds could be partly responsible for the deeper solsticial pause produced in $\tau_{M Y 24}^{*}$, at least in late autumn $\left(L_{s}=240-270^{\circ}\right)$.

\subsection{Topographic heterogeneity}

With or without ice clouds, winter midlatitude and polar dynamics are undoubtedly influenced significantly by the substantial and complex topography that exists on Mars. Fig. 13 shows the difference between the zonal mean martian surface and the areoid (the martian equivalent of terrestrial sea level), and the standard deviation of the surface height in the zonal direction. Most of the northern hemisphere slopes downward towards the pole, while the southern polar region slopes upward towards the pole. Blumsack and Gierasch (1972) suggested that the northern hemispheric surface, with the lower boundary sloping in the opposite sense to atmospheric isentropes, should reduce baroclinic instability and shift the most unstable mode to shorter wavelengths, with the magnitude of the effect increasing with the steepness of the gradient. The meridional topographic slope is steeper over $35-50^{\circ} \mathrm{N}$ than it is over $50-75^{\circ} \mathrm{N}$, which would suggest that transient eddy activity in the northern hemisphere should be reduced if and when the baroclinic zone moves south of ${ }_{495} \sim 50^{\circ} \mathrm{N}$. Using the temperature gradients of Fig. 3(d) as a guide, this progres- 


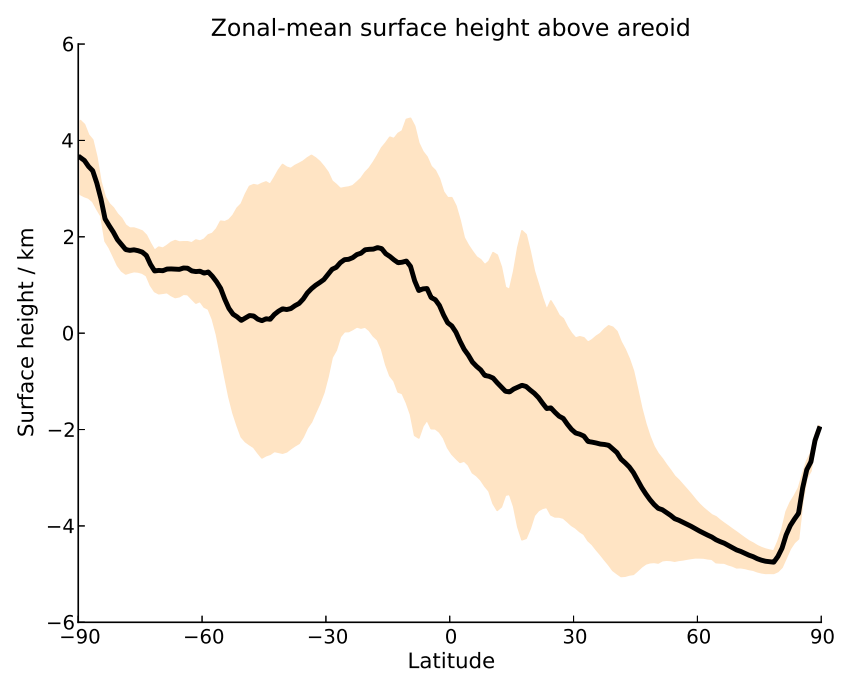

Fig. 13. Height of the zonally averaged martian surface above the areoid $(\mathrm{km}$, solid line). Shading (mean $\pm 1 \sigma$ ) indicates standard deviation in surface height around latitude circles.

sion to or beyond $50^{\circ} \mathrm{N}$ appears to coincide with the duration of the solsticial pause. A similar argument could be made for the southern hemisphere in the region north of $50^{\circ} \mathrm{S}$, as the slope changes direction, although the meridional variation is more complicated than it is in the northern hemisphere.

The martian surface is also notably more zonally asymmetric in midlatitudes than it is poleward of $60^{\circ}$ (note the varying spread in Fig. 13). Yu and Hartmann (1995) found that adding topographic features to a zonally symmetric surface results in a transfer of wave energy from transient to stationary waves, and from high to low frequencies. In their paper, 'high frequency' was defined as those waves with a period of less than 7 days: therefore, it is possible that most martian transient waves, having relatively short periods, will be suppressed to some extent by the presence of the large zonal variations in topography that exist equatorward of $60^{\circ}$. 
A critical latitude for suppression of instability can be hypothesised, situated at around $50-60^{\circ}$ in each hemisphere. Since the seasonal ice caps grow beyond this latitude around midwinter, a topographic contribution to the seasonal variation of eddy activity is possible, caused by one or both of the increase in meridional slope and the increase in zonal asymmetry equatorward of $60^{\circ}$. To investigate which, if either, of these effects exert an influence, two further model runs were performed. One simulation, zonav_ $\tau_{M Y 24}^{*}$, used zonally averaged topography, while the other, zmrem_ $\tau_{M Y 24}^{*}$, had the mean meridional slope of the topography removed by subtracting the zonal mean surface height from each gridpoint. Both were forced by the MY24 dust scenario and included radiatively active water ice clouds. The dust scenario was not zonally or meridionally smoothed; however, the scenario is defined relative to a reference pressure level, so dust opacities do not retain a signal of the non-averaged martian surface, only of heterogeneities that existed in the opacity field at the reference level.

The results, which can be compared to those in Fig. 3(d), are shown in Fig. 14. In zonav_ $\tau_{M Y 24}^{*}$, transient temperature perturbations in the northern hemisphere are increased over those from $\tau_{M Y 24}^{*}$ throughout autumn and winter, and a solsticial pause is no longer produced; in fact, there is now a solsticial maximum. This is accompanied by a consistently large vertical wind shear (and therefore large baroclinic growth rate) at $60-75^{\circ} \mathrm{N}$, and a secondary maximum at $10-30^{\circ} \mathrm{N}$ (not shown). The region of peak temperature variance spreads further south, as far as $30-40^{\circ} \mathrm{N}$, where previously there was a strong $s=3$ topographic forcing.

The difference in solsticial pause depth between $\tau_{M Y 24}$ and $\tau_{M Y 24}^{*}$ can, therefore, be at least partially attributed to the influence of the clouds in shifting 

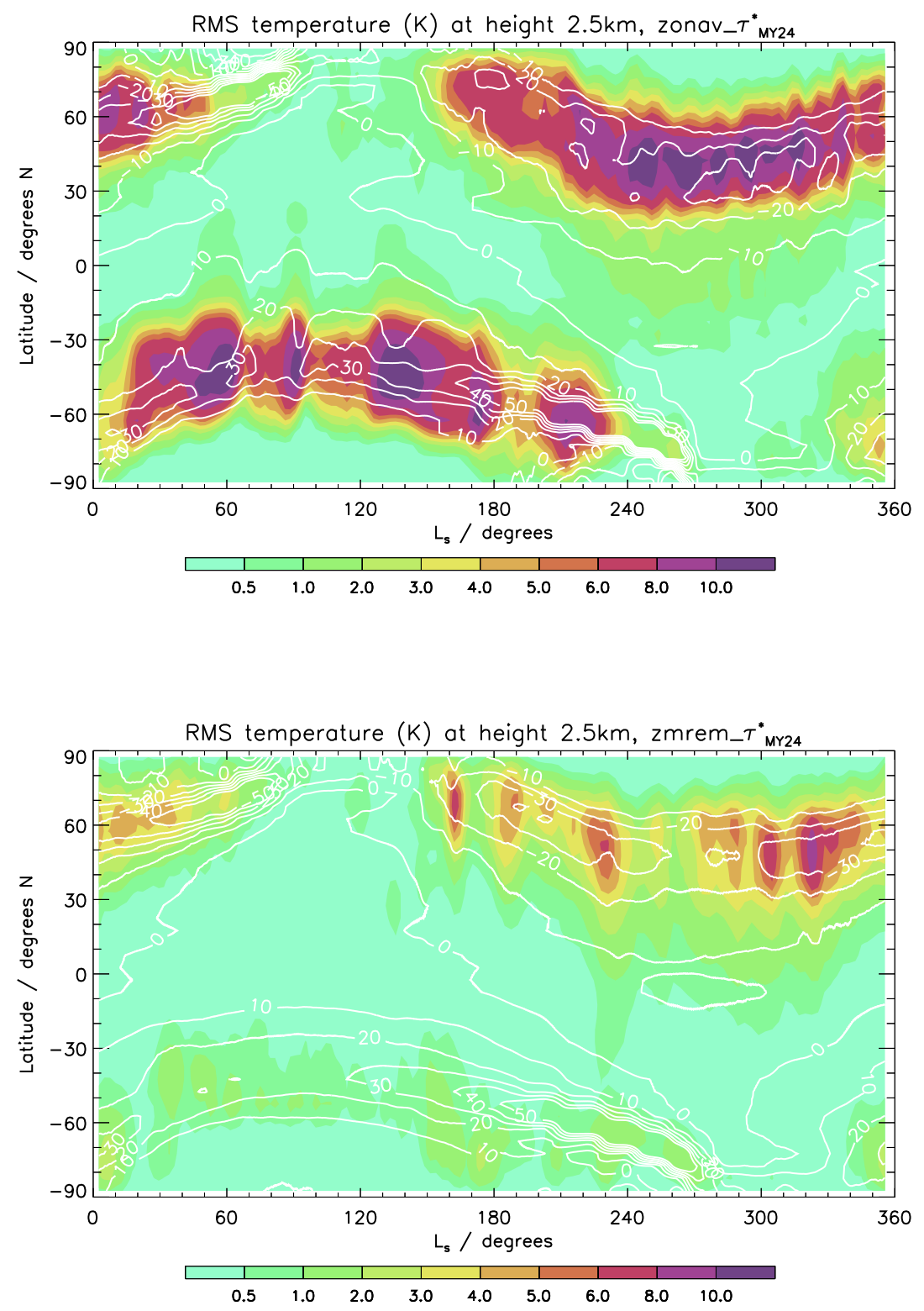

Fig. 14. Transient RMS temperature (K) at $2.5 \mathrm{~km}$ for runs using the MY24 dust scenario with radiatively active water ice clouds, and using zonally averaged topography (zonav_ $\tau_{M Y 24}^{*}$, top), and topography with the mean meridional slope removed (zmrem_ $\tau_{M Y 24}^{*}$, bottom). The meridional temperature gradient at $200 \mathrm{~m}$ $\left(10^{-6} \mathrm{~K} \mathrm{~m}^{-1}\right)$ is overlaid in white contours. 
the region of peak eddy activity equatorwards and further into the topographically asymmetric midlatitudes, where eddy growth seems to be impeded.

A dramatic change is seen in the southern hemisphere, where peak RMS temperatures increase and are now of comparable magnitude to those in the northern hemisphere. Several short maxima and minima are seen but transient eddies remain fairly strong over $L_{s}=30-150^{\circ}$ at $30-50^{\circ} \mathrm{S}$, whereas in $\tau_{M Y 24}^{*}$ they are largely absent from this region/season. It appears that, in reality, the zonal topographic asymmetry at these latitudes (which is predominantly of wavenumbers 1 and 3) is a major reason for the weakness of eddy activity in the southern hemisphere, where RMS values $\gtrsim 4 \mathrm{~K}$ are rarely seen north of $60^{\circ} \mathrm{S}$. In this simulation with zonally averaged topography, peak Eady growth rates at $200 \mathrm{~m}$ at $60-70^{\circ} \mathrm{S}$ (not shown) are much larger than for $\tau_{M Y 24}^{*}$, though in fact growth rates are increased throughout $40-80^{\circ} \mathrm{S}$, and peaks in RMS temperature appear to follow fluctuations in local growth rate at $\sim 45^{\circ} \mathrm{S}$ for much of the autumn/winter period.

The simulation zmrem_ $\tau_{M Y 24}^{*}$ produces a marginally shallower minimum in the northern hemisphere than $\tau_{M Y 24}^{*}$, implying that an increased meridional slope at $35-50^{\circ} \mathrm{N}$ does (in $\tau_{M Y 24}^{*}$ ) slightly suppress instability. More notably, however, there is a general reduction in temperature variance in the northern hemisphere: with the exception of the pre-solstice peak, Eady growth rates are larger than they are in $\tau_{M Y 24}^{*}$, yet weaker waves result throughout autumn and winter. Increased growth rates are consistent with the removal of a lower boundary that slopes downwards towards the pole, but these do not result in stronger eddies, possibly a result of the decreased surface pressure in the northern hemisphere, as the surface now sits higher relative to the areoid than in $\tau_{M Y 24}^{*}$, which implies a lower $\mathrm{CO}_{2}$ frost point and greater temperature 
inversions over the cold polar cap, making the atmosphere more statically stable. It would appear that this has a greater effect on transient eddies than does the change to the slope of the lower boundary.

The southern hemisphere sees very little change from $\tau_{M Y 24}^{*}$, suggesting a greater degree of balance between the effects of changing surface pressure and changing meridional slope.

\section{Discussion}

\subsection{The role of topography}

From comparing the zonally averaged topography simulation zonav_ $\tau_{M Y 24}^{*}$ to those described in earlier sections, it is clear that the zonal asymmetry of the midlatitude topography exerts a very strong influence on transient activity in both hemispheres. In the case of the southern hemisphere, the removal of this asymmetry increases model RMS temperature during autumn and winter by around a factor of two, bringing magnitudes close to those of the northern hemisphere, and implying that, although winter baroclinicity is slightly weaker in the southern hemisphere on account of Mars's orbital eccentricity, it is in fact the topographic zonal asymmetry that is primarily responsible for the relatively weak transient eddies observed in southern autumn and winter.

In the northern hemisphere, the absence of zonal variations in topography allows a large surface vertical wind shear to be maintained throughout autumn and winter at $60-70^{\circ} \mathrm{N}$, where it would otherwise tend to reduce around solstice, as the polar ice cap edge extends into the more zonally heteroge- 
neous midlatitude region. A secondary peak in wind shear and growth rate at $10-30^{\circ} \mathrm{N}$ is possibly also important in creating the solsticial maximum seen in zonav_ $\tau_{M Y 24}^{*}$. These results suggest that while a decrease in atmospheric baroclinicity close to the surface is the direct explanation for the northern hemisphere solsticial pause, this arises more fundamentally due to the existence of zonally asymmetric topography in the midlatitudes.

Additional altered-topography runs could help to determine why the zonal asymmetry in topography suppresses waves before and after solstice so much more strongly in the southern hemisphere than in the northern hemisphere. The amplitudes of the zonal topographic variability are fairly similar in each of the two hemispheres, but perhaps crucially the zonal standard deviation is larger at around $50^{\circ} \mathrm{S}$ than at $50^{\circ} \mathrm{N}$ (Fig. 13). It may be necessary to look 595 at the longitudinal patterns of energy generation, as in Kavulich Jr et al. (2013), rather than using zonal-mean diagnostics, to understand the specific impacts of topographic features in both hemispheres. This issue is pertinent to the occurrence of cap-edge dust storms (and potentially also larger storms) during the recent history of Mars, since the precession of the solar longitude of perihelion, which occurs with a period of $\sim 50 \mathrm{kyr}$ (Ward, 1974), is roughly equivalent to the flipping of the topography about the equator every $\sim 25 \mathrm{kyr}$.

Further study of the zonal distribution of martian eddy activity could also reveal the similarities and differences that exist between the roles of topography in the solsticial pause and in the terrestrial midwinter suppression. In the latter case, interactions between the surface and low-level flow in one specific region, central Asia, have been suggested to reduce local baroclinic conversion so as to create a downstream solsticial minimum in eddy activity over the North Pacific in some years (Penny et al., 2010; Park et al., 2010). On Mars, 
the reduction in eddy activity occurs to a similar degree at all longitudes (see

P1, Figs. 7 \& 8), suggesting that an 'upstream seeding' model may not be appropriate. However, there may exist similarities between the martian situation and the seasonal variation in the eddy energy generation process that occurs over the central Asian mountains.

\subsection{Model dependence}

The effects of dust, ice cloud and topographic forcings have been diagnosed above in the UKMGCM, but it must be noted that other models show some variation in simulated solsticial pause depth. For example, Wang et al. (2013) show a pause of comparable magnitude to that of $\tau_{M Y 24}^{*}$, from a simulation without radiatively active ice clouds and with a moderate increase in dust opacity near solstice. Basu et al. (2006) also present a plausible solsticial pause, though it is unclear what dust loading was used for this simulation. It may be that the dynamics of these models differ from those of the UKMGCM, such that they simulate a sharper drop-off in near-surface vertical wind shear around solstice in a cloud-free run. Indeed, differences have been seen between the two versions of the LMD/UK model (using either the LMD or UK dynamical core), under the same dust forcing.

Supporting the idea that the presence of water ice clouds contributes to the formation of the solsticial pause is the modelling work of Wilson (2011), who also simulated a deep solsticial pause only when radiatively active water ice clouds were included. The addition of active clouds to the other models mentioned above can therefore be expected to increase the depth of the simulated pauses, which would bring them even closer to observations. However, the 


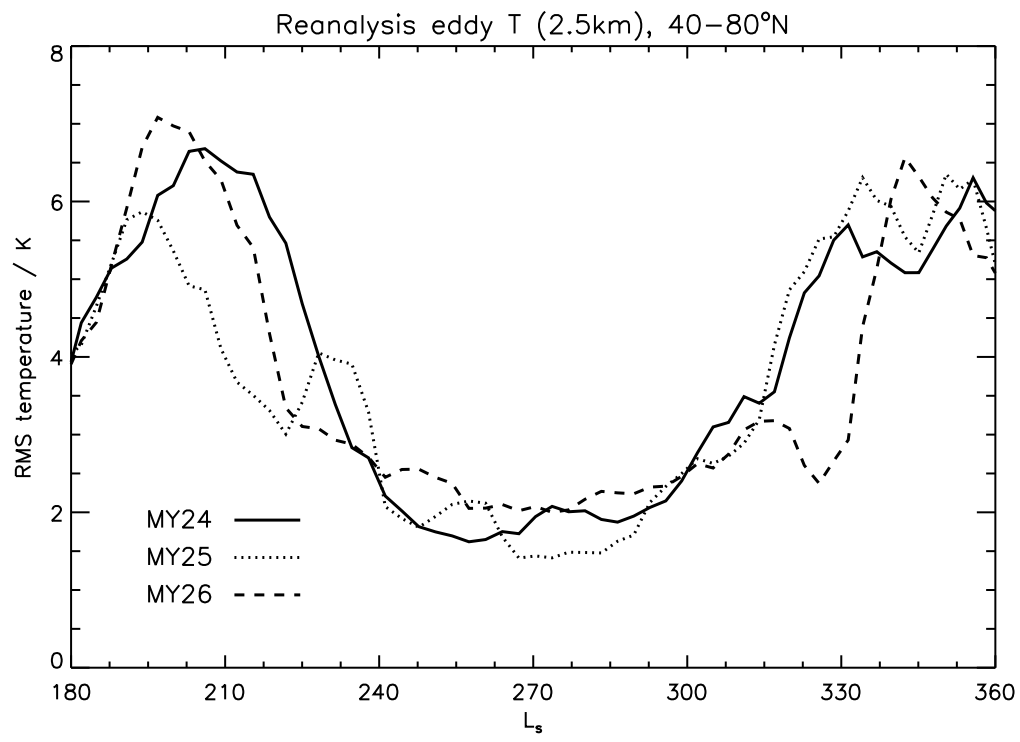

Fig. 15. Transient RMS temperature at $2.5 \mathrm{~km}$, averaged between $40^{\circ} \mathrm{N}$ and $80^{\circ} \mathrm{N}$, for MY24 (solid), MY25 (dotted) and MY26 (dashed), from the reanalysis.

magnitude of the impact of ice clouds may differ in other models, due to their stronger cloud-free simulated solsticial pauses.

\subsection{Interannual variability in near-surface travelling waves}

Our insight into the mechanisms behind the suppression of near-surface eddies in northern midlatitudes allows us to draw some conclusions regarding the interannual variability seen in northern hemispheric travelling waves in the TES reanalysis data, building on ideas discussed in Wang (2007). The three years of transient eddy temperature data from the reanalysis can be seen in Figs. 1 and 2 of P1; here, Fig. 15 illustrates more clearly the variation in the length and depth of the northern hemisphere solsticial pause at the surface.

The earlier reduction in transient eddy activity seen in MY26 compared to MY24 followed the earlier development of the upper maximum and tilt of 
the westerly jet due to the regional storm at $L_{s}=210^{\circ}$ in MY26. Wang (2007) found that flushing storms, which disappeared upon the onset of the $210^{\circ}$ storm, did return briefly over $L_{s}=230-250^{\circ}$ which, as can be seen from Fig. 1 of P1, was a period of moderate transient activity along the cap edge. She attributed the eventual cessation of flushing storms at $L_{s}=250^{\circ}$ to the progression into the solsticial window, where the change in atmospheric state makes it impossible to sustain flushing storm activity. The particular aspect of this change that is relevant, according to the results shown here in previous sections, is the tilt of the westerly jet, particularly near the ground, which increases naturally around solstice, even without a substantial increase in global dust loading (Figs. 5, 6).

Conversely, a large dust storm may not fully suppress surface transient eddies if it occurs too early in the year. The pre-solstice peak in surface RMS temperature was lower in MY25 than in the other two years, but nevertheless values of $\sim 6 \mathrm{~K}$ were present at $L_{s}=200^{\circ}$, while the global dust storm was in progress. It seems that pre- $210^{\circ}$ is simply too early to force a sufficient tilt in the jet near the ground to fully suppress surface instability, even with a storm as large as the one in MY25. This fits with the results of simulation $\tau_{\text {high }}$ (Fig. 9), in which eddy activity was not reduced until after $L_{s} \approx 220^{\circ}$, despite an equally high dust loading during $L_{s}=180-220^{\circ}$. pared to the previous two years: this can be linked to the dramatic increase in dust loading seen towards the end of that year (the $L_{s}=315^{\circ}$ dust storm), which led to a temporary reversal of the seasonal decrease in the magnitude of the polar warming, and associated tilt of the jet, that usually occurs at this time of year. When the late-winter storm occurred, the temperature at 
$\left(10 \mathrm{~Pa}, 60^{\circ} \mathrm{N}\right)$, which had been declining since $L_{s}=270^{\circ}$, increased again, by $\sim 10 \mathrm{~K}$, as the PMOC expanded in response to the increase in dust loading. The tilt that this imparted to the jet allowed a renewed suppression of the near-surface vertical wind shear and therefore of the transient eddy activity, which had been increasing in line with the other two years until the occurrence of the storm.

Dust lifting in the northern midlatitudes therefore appears to possess a selflimiting quality, whereby the occurrence of a series of flushing storms is likely to inhibit the development of further such storms through the strengthening of the PMOC (Wang, 2007). Periods of flushing storm activity in early autumn and late winter seem certain to occur, however, because outside $L_{s} \approx 210-330^{\circ}$ it is very difficult to force the tilt in the lower part of the westerly jet that is needed to reduce near-surface growth rates. What is less certain is that there must always be a window where no flushing storms occur. Whether or not these conditions occur in a particular year may depend on there being a sufficiently large dust loading around northern winter solstice, resulting either from the occurrence of a sequence of flushing storms or more simply by an increase to the 'background' loading above some baseline level. The examination of further Mars years recently added to the reanalysis (Montabone et al., 2015) has the potential to shed further light on this matter.

Although no such increased dust loading can be invoked to explain the annual pause in the southern hemisphere, it is possible that the global circulation is altered in an equivalent way by the presence of the thick tropical cloud belt around solstice (Madeleine et al., 2012), affecting the tilt of the southern winter jet. This would explain the presence of a southern hemisphere solsticial pause in $\tau_{\text {low }}^{*}$ (Fig. 3(b)) but not in $\tau_{\text {low }}$ (Fig. 3(a)). 


\section{Conclusions}

The martian solsticial pause, as presented in P1, has been convincingly simulated by the UKMGCM, when run with a realistic dust loading and the inclusion of radiatively active water ice clouds. Both increased dustiness and ice clouds alter the thermal structure of the polar atmosphere, decreasing the zonal wind and its vertical shear near the surface at mid- and high latitudes around winter solstice, causing a decrease in baroclinic growth rates.

Baroclinic eddy activity near the surface in both hemispheres is reasonably well predicted by linear instability theory. In all model runs that produce a solsticial pause, and to a lesser extent in the reanalysis of MY24, variation in transient eddy amplitudes is accompanied by variation in Eady growth rate, driven by changes in near-surface vertical wind shear as well as in the background state $(f, N)$. Quantitative agreement was found between variations in growth rate and the amplitude of temperature perturbations, though in general it is necessary to consider the growth rate at $60-70^{\circ}$ latitude rather than at the location of the eddy maximum, in midlatitudes. There is evidence that a high wind shear at these high latitudes can sustain eddy activity at midlatitudes, though the effect was seen not to apply in the southern hemisphere, perhaps due to different meridional flow conditions near the surface.

The barotropic governor effect was found not to play a significant role in the martian solsticial pause in either hemisphere. Diabatic dissipation, in the form of radiative damping, may play a modest role, in concert with modulations in the local Eady growth rate itself. Zonal variations in midlatitude topography exert a strong control on near-surface transient eddies, reducing their ampli- 
tude dramatically in the southern hemisphere throughout autumn and winter, and around winter solstice in the northern hemisphere. It appears, therefore, that the zonal topographic asymmetry in midlatitudes is the ultimate cause of the solsticial pause in both hemispheres.

\section{Acknowledgements}

The authors thank Don Banfield and an anonymous reviewer for their careful and helpful reviews. We acknowledge useful discussions with John Wilson and Luca Montabone. This work was funded by the UK Science and Technology Facilities Council.

\section{References}

J. R. Barnes, J. B. Pollack, R. M. Haberle, C. B. Leovy, R. W. Zurek, H. Lee, and J. Schaeffer. Mars atmospheric dynamics as simulated by the NASA AMES General Circulation Model. II - Transient baroclinic eddies. J. Geophys. Res., 98:3125-3148, feb 1993. doi: 10.1029/92JE02935.

S. Basu, J. Wilson, M. Richardson, and A. Ingersoll. Simulation of spontaneous and variable global dust storms with the GFDL Mars GCM. J. Geophys. Res. (Planets), 111(E10):9004-+, sep 2006. doi: 10.1029/2005JE002660.

J. L. Benson, D. M. Kass, and A. Kleinböhl. Mars' north polar hood as observed by the Mars Climate Sounder. J. Geophys. Res. (Planets), 116 (E15):E03008, mar 2011. doi: 10.1029/2010JE003693.

S. L. Blumsack and P. J. Gierasch. Mars: The effects of topography on baro- 
clinic instability. J. Atmos. Sci., 29(6):1081-1089, 1972. doi: 10.1175/15200469(1972)029<1081:MTEOTO>2.0.CO;2.

E. K. M. Chang. GCM and Observational Diagnoses of the Seasonal and Interannual Variations of the Pacific Storm Track during the Cool Season. J. Atmos. Sci., 58:1784-1800, jul 2001. doi: 10.1175/15200469(2001)058<1784:GAODOT >2.0.CO;2.

Y. Deng and M. Mak. Nature of the Differences in the Intraseasonal Variability of the Pacific and Atlantic Storm Tracks: A Diagnostic Study. J. Atmos. Sci., 63:2602-2615, oct 2006. doi: 10.1175/JAS3749.1.

F. Forget, F. Hourdin, R. Fournier, C. Hourdin, O. Talagrand, M. Collins, S. R. Lewis, P. L. Read, and J.-P. Huot. Improved general circulation models of the Martian atmosphere from the surface to above $80 \mathrm{~km}$. J. Geophys. Res., 104:24155-24176, oct 1999. doi: 10.1029/1999JE001025.

F. Hourdin, F. Forget, and O. Talagrand. The sensitivity of the Martian surface pressure and atmospheric mass budget to various parameters: A comparison between numerical simulations and Viking observations. J. Geophys. Res., 100:5501-5523, mar 1995. doi: 10.1029/94JE03079.

I. N. James and L. J. Gray. Concerning the effect of surface drag on the circulation of a baroclinic planetary atmosphere. Q. J. Roy. Meteor. Soc., 112:1231-1250, oct 1986. doi: 10.1002/qj.49711247417.

M. J. Kavulich Jr, I. Szunyogh, G. Gyarmati, and R. J. Wilson. Local dynamics of baroclinic waves in the Martian atmosphere. J. Atmos. Sci., 70 (11):3415-3447, 2013.

T. Kuroda, A. S. Medvedev, P. Hartogh, and M. Takahashi. Seasonal changes of the baroclinic wave activity in the northern hemisphere of Mars simulated with a GCM. Geophys. Rev. Lett., 34:L09203, may 2007. doi: 10.1029/2006GL028816. 
S. R. Lewis, D. P. Mulholland, P. L. Read, L. Montabone, R. J. Wilson, and M. D. Smith. The solsticial pause on Mars: 1 A planetary wave reanalysis. Icarus, this issue.

J.-B. Madeleine, F. Forget, E. Millour, L. Montabone, and M. J. Wolff. Revisiting the radiative impact of dust on Mars using the LMD Global Climate Model. J. Geophys. Res. (Planets), 116:E11010, November 2011. doi: 10.1029/2011JE003855.

J.-B. Madeleine, F. Forget, E. Millour, T. Navarro, and A. Spiga. The influence of radiatively active water ice clouds on the martian climate. Geophys Res Lett, 39(23):n/a-n/a, 2012. ISSN 1944-8007. doi: 10.1029/2012GL053564.

D. J. McCleese, J. T. Schofield, F. W. Taylor, S. B. Calcutt, M. C. Foote, D. M. Kass, C. B. Leovy, D. A. Paige, P. L. Read, and R. W. Zurek. Mars Climate Sounder: An investigation of thermal and water vapor structure, dust and condensate distributions in the atmosphere, and energy balance of the polar regions. J. Geophys. Res. (Planets), 112(E11):5-+, may 2007. doi: 10.1029/2006JE002790.

L. Montabone, F. Forget, E. Millour, R. J. Wilson, S. R. Lewis, B. Cantor, D. Kass, A. Kleinböhl, M. T. Lemmon, M. D. Smith, and M. J. Wolff. Eightyear climatology of dust optical depth on Mars. Icarus, 251(0):65-95, 2015. ISSN 0019-1035. doi: 10.1016/j.icarus.2014.12.034.

F. Montmessin, F. Forget, P. Rannou, M. Cabane, and R. M. Haberle. Origin and role of water ice clouds in the Martian water cycle as inferred from a general circulation model. J. Geophys. Res. (Planets), 109(E18):10004-+, oct 2004. doi: 10.1029/2004JE002284.

H.-S. Park, J. C. H. Chiang, and S.-W. Son. The Role of the Central Asian Mountains on the Midwinter Suppression of North Pacific Storminess. J. Atmos. Sci., 67:3706-3720, nov 2010. doi: 10.1175/2010JAS3349.1. 
J. P. Peixoto and A. H. Oort. Physics of climate. New York: American Institute of Physics (AIP), 1992.

S. Penny, G. H. Roe, and D. S. Battisti. The Source of the Midwinter Suppression in Storminess over the North Pacific. J. Climate, 23:634-+, 2010. and observation, pages 147-150, February 2011. 
J.-Y. Yu and D. L. Hartmann. Orographic Influences on the Distribution and Generation of Atmospheric Variability in a GCM. J. Atmos. Sci., 52:2428825 2443, jul 1995. doi: 10.1175/1520-0469(1995)052<2428:OIOTDA > 2.0.CO;2. 